\title{
Constitutive modeling of the mechanics associated with triple shape memory polymers
}

\author{
S. Moon ${ }^{\text {a }}$, F. Cui ${ }^{\mathrm{a}}$ and I. J. Rao ${ }^{\mathrm{a}, *}$ \\ a Department of Mechanical Engineering, New Jersey Institute of Technology, Newark, NJ \\ 07012, United States
}

*Corresponding Author: I. J. Rao, Department of Mechanical and Industrial Engineering, NJIT, Newark, NJ 07102. e-mail: raoi@njit.edu. Phone: 001-973-596-5601. Fax: 001-973-642-4282 


\begin{abstract}
Shape memory polymers (SMPs) are smart materials that alter their shape in response to external stimuli. Dual-shape SMPs are widely recognized and are characterized by two shapes involved in a typical shape memory cycle. Recently, triple-shape memory polymers (TSMPs) have been introduced that have the ability to remember three shapes. Thermo-sensitive TSMPs can perform two sequential shape changes in response to heat, which were programed previously in a triple-shape creation process (TSCP). TSMPs are technologically significant as their development of has led to emergence of many complex potential applications that cannot be achieved by dual-shape polymers [54]. Crystallizable TSMPs are a class of thermo-sensitive TSMPs, where the shape creation is due to formation of crystalline phases. Different TSCPs have been reported which enable to control the triple shape capability of these materials. In this work the mechanical behavior of TSMPs has been modeled using a framework that has been developed recently for studying crystallization in polymers ([28], [25], [27], [31]). The framework has been used successfully to model SMPs ([43], [44]), light activated SMPs ([49]) and is based upon the theory of multiple natural configurations. The model has been used to simulate a uni-axial deformation cycle for different types of TSCPs and the results have been compared with experimental data.
\end{abstract}

Keywords: Shape memory; Polymers; Triple-shape effect; Triple-shape creation procedures; multiple natural configurations

\title{
1. Introduction
}

Smart materials are actively moving materials that can alter their shape in a controlled manner on demand, in reaction to an external stimulus. Shape memory polymers (SMPs) belong to a class of these technologically significant smart materials that have attracted tremendous scientific interest and have undergone explosive growth within the last decade. SMPs have the capacity to perform changes in their shape in a predefined way when activated by use of various forms of external stimuli $[4,8$, and 9]. The most commonly used stimulus is heat. SMPs are similar to shape memory alloys (SMAs); however, compared to SMAs they possess many advantages. Some of the advantages of SMPs are, they have the ability to undergo large deformation during the shape memory cycle, ease of processing, low cost, and ease of tailoring the thermo-mechanical properties according to application involved. Conventionally, a SMP can fix one transient shape even after undergoing significant deformation from a permanent shape. Recovery of the original shape is triggered by exposure to an external stimulus. This shape memory effect is known as "a dual shape memory effect" as there are two shapes involved in each shape memory cycle. Recently, Triple-shape memory materials have been introduced that have the capability to change from a second transient shape to a first transient shape and from there to the original shape, when stimulated by external stimuli such as heat $[40,41,42,45,46,47,50,51,52,53,54$ and 11]. These thermal transitions associated with the polymer segments can be glass transition or melting points. The fields of application in which SMPs are finding use are highly diverse and cover a broad range. These include packaging, electronics, textiles and extend to sophisticated biomedical or aerospace applications. Some specific examples are packaging (e.g. heat-shrinkable foils/tubes), textiles (e.g. adjustable appearance or controlling water permeation ), active assembly and electronics (e.g. actuators) to sophisticated aerospace (e.g. morphing structures) and biomedical applications (e.g. shape expandable implants for minimally invasive surgery) $[11,49,35,14,16,33,48,36,50,51,52,53,54,55,56]$. An important subclass of SMPs are crystallizable SMPs (CSMPs), wherein entanglement of the molecules or 
crosslinks are commonly used to remember the permanent shape, and formation of the crystalline phase is used to retain the transient shape, with polymer returning to its original shape on melting of the crystalline phase. Properties of CSMPs can be easily adjusted depending on the application (see [32, 5, 10, and 39]). In crystallizable triple shape memory polymers (TSMPs) the formation of two subsequent crystalline phases is used to retain two transient shapes. The polymer returns from the second temporary shape to the first temporary shape and from there to the permanent shape on subsequent melting of the crystalline phases due to heating (see [40,41,46, and 47]). This paper exclusively focuses on crystallizable triple shape memory polymers which we denote by TSMP.

TSMPs can be either thermosets or thermoplastics. Thermoset polymer networks in their rubbery state consist of covalently cross-linked flexible polymer chains and shape of the polymer is defined by the covalent crosslinks. In thermoplastic TSMPs the permanent shape is defined by physical cross-links. TSMPs have the ability to form two different crystalline domains at two different temperatures that can fix two shapes. One of the switching domains has a higher transition temperature than the other. If the polymer is cooled from a temperature $T_{H}$ that is above both the transition temperatures, to a temperature $T_{\text {mid }}$ that is below the first transition temperature denoted here by $T_{R_{1}}\left(T_{\text {mid }}<T_{R_{1}}<T_{H}\right)$ the first domain or phase- 1 is solidified. If the material is cooled while it is in a deformed configuration, the crystallites are formed in the deformed configuration, and they act as temporary crosslinks that prevent the TSMP from returning to its original shape. This leads to the fixing of the first temporary shape (B). On unloading the specimen below $T_{R_{1}}$ a small amount of recovery is observed as the polymer has its original crosslinks still in place. Similarly when the polymer is cooled to a temperature $T_{L}$ that is below the second transition temperature denoted here by $T_{R_{2}}\left(T_{L}<T_{R_{2}}<T_{\text {mid }}<T_{R_{1}}<T_{H}\right)$ the second domain or phase-2 is solidified. During the second cooling process some part of the first domain or phase-1 also solidifies along with the second domain. If the material is cooled while it is in the deformed configuration, these crystallites are formed in the deformed configuration, and they act as temporary crosslinks that prevent the TSMP from returning to the first temporary shape, this leads to the fixation of the second temporary shape (A). On unloading the specimen below $T_{R_{2}}$ a small amount of recovery is observed as the polymer has its original crosslinks, and the temporary crosslinks formed during the first cooling process still in place. On subsequent heating above $T_{R_{2}}$ the crystalline phase associated with the second domain or phase-2 melts and the TSMP returns from the second temporary shape (A) to the first temporary shape (B). On further heating to a temperature above $T_{R_{1}}$ the crystalline phase associated with the first domain or phase- 1 that is formed both at the higher temperature and the lower temperature melts and the TSMP returns to its original shape $(\mathrm{C})$. The orientation of the crystals formed below recovery temperature depends on the deformation undergone by the polymer just prior to cooling (see [39]). These crystals will have a preferred direction depending on the deformation that will cause material properties to be anisotropic

The shape memory behavior of a TSMP is illustrated schematically in Fig.1 along with a typical uniaxial stress-strain curve, Fig.2, (see [41], [42]). Here, it should be noted that other cycles are possible depending on how the TSMP is created, which we will discuss later in the paper. In Fig.1 the filled circles represent the cross-links (they could be chemical or physical cross-links), the wavy line connecting 
the cross-links represents the crystallizable polymer in its amorphous state while the rectangular blocks represent the first domain in a crystalline state. The triangular blocks represent the second domain in a crystalline state. State 1 in both figures denotes the undeformed configuration. Above both the recovery temperatures $T_{R_{1}}$ and $T_{R_{2}}$ the polymer behavior is rubber-like and its elastic behavior is driven by changes in entropy. On deforming above $T_{R_{1}}$ the recovery temperature associated with the first domain the molecules between the cross-links stretch (state 2). If the polymer is now cooled to a temperature below $T_{R_{1}}$, crystallization of the first domain takes place and the crystals are formed in this deformed configuration. If stretch is held constant the onset of crystallization is accompanied by a sharp drop in stress (from state 2 to state 3 ). After unloading (state 3 to state 4 ) the polymer remains in a deformed configuration with a small amount of recovery. This recovery is due to the presence of two components (amorphous and crystalline domain-1) each with their own stress-free states. The amorphous part has a tendency to retract to its original configuration while the crystalline part prefers the deformed configuration that it was formed in. As the crystalline part is a lot stiffer, the recovery strain is small (see Fig.2). On deforming above the recovery temperature of the second domain $T_{R_{2}}$, the polymer molecules between the original cross-links (physical/chemical) and the temporary cross-links formed after the first cooling process stretch (state 5). If the polymer is now cooled to a temperature below $T_{R_{2}}$, crystallization of the second domain takes place. During this second cooling process, along with the second domain, some part of the first domain also crystallizes. Again, the onset of crystallization is accompanied by a sharp drop in the stress (from state 5 to state 6). After unloading (state 6 to state 7 ) the polymer remains in a deformed configuration with a small amount of recovery. This recovery is due to the presence of three components amorphous, crystalline domain-1 or phase-1 formed both at the higher and lower temperatures, crystalline domain-2 or phase- 2 each with their own stress-free states. The amorphous part has a tendency to retract to its original configuration while the crystalline parts prefer the deformed configurations where they were formed. As the crystalline part is a lot stiffer the recovery strain is small (see Fig.2). The mechanical response of the polymer in this state is similar to that of a semi-crystalline polymer with oriented crystallites i.e., it is relatively stiff and the mechanical behavior is anisotropic. Usually when this semi-crystalline polymer is subjected to small deformations it exhibits elastic behavior, energetic in origin. When the polymer is heated to above $T_{R_{2}}$, (from state 7 to state 4 ) the crystallites associated with the second domain melt and the polymer retracts to its first temporary shape. On further heating to above $T_{R_{1}}$, (from state 4 to state 1 ) the crystallites associated with the first domain that were 


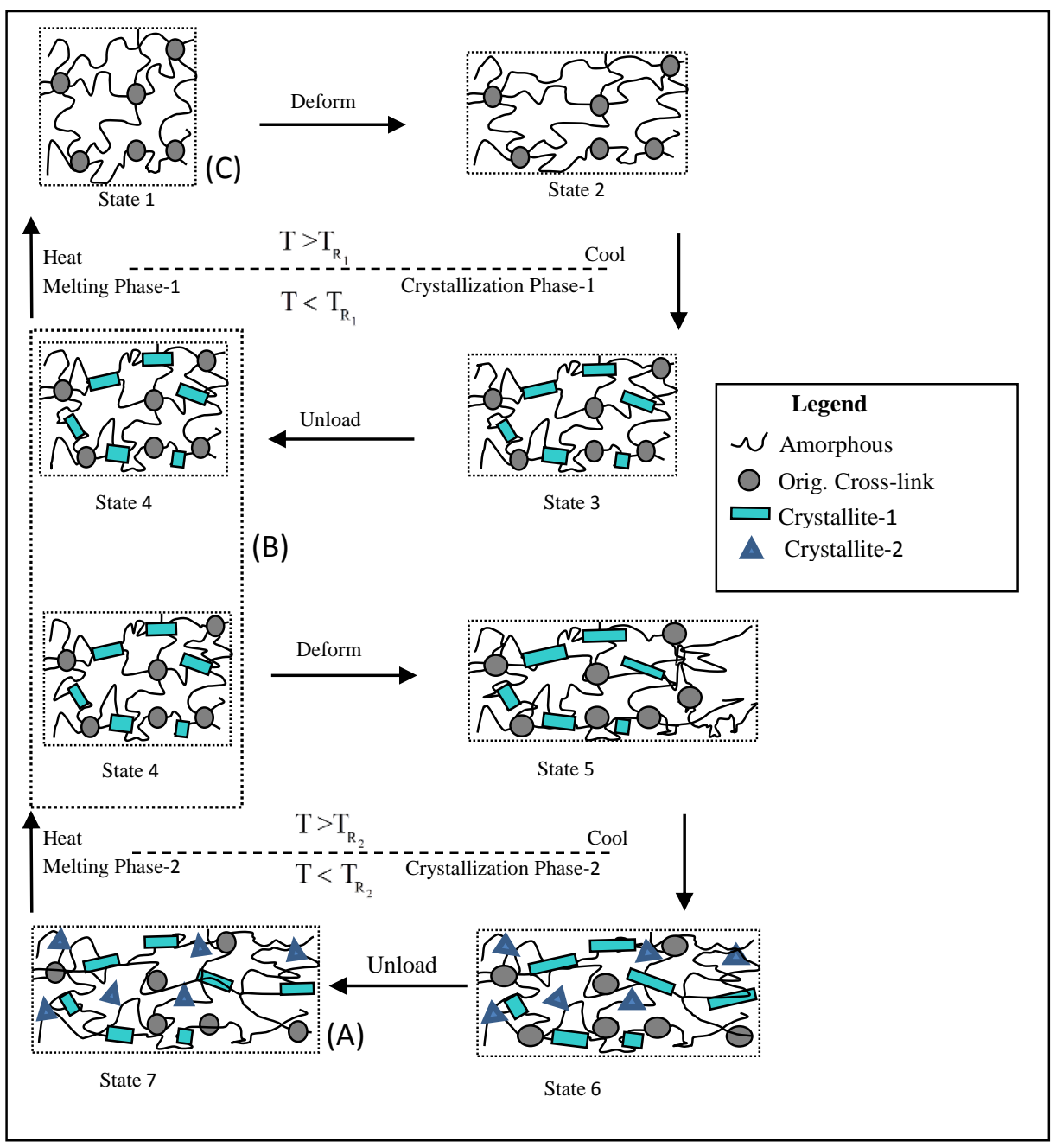

Figure 1. Schematic illustration of the Triple shape effect in TSMPs in a typical triple shape memory creation process. (A - Second temporary shape, B - First temporary shape and C - Permanent shape).

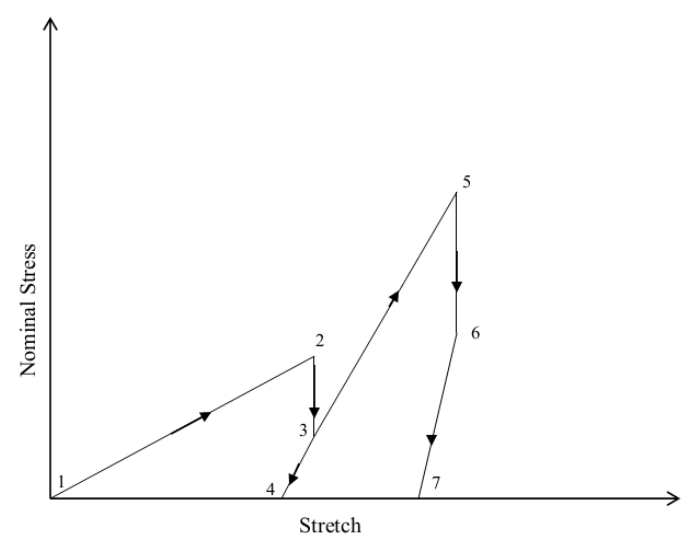

Figure 2. A typical stress versus stretch curve of a TSMP for the deformation cycle illustrated in Fig 1. 
formed both at a higher temperature and a lower temperature melt returning to their original amorphous state, and the polymer retracts to its original shape.

As we can see from the above discussion, in order to model the thermodynamic behavior of the TSMPs we need to characterize their behavior above, below and in between the recovery temperatures $T_{R_{1}}$ and $T_{R_{2}}$. This includes the processes of crystallization at the higher and the lower temperatures and the two melting processes. Above both the recovery temperatures the polymer behaves like a rubber. When cooled to below their crystallization temperatures, the crystallizable part of the respective domains crystallizes. The orientation of the crystals depends on the deformation of the polymer during the crystallization process and in turn the orientation of the crystals is a key element in determining the mechanical properties of this semi-crystalline polymer, for instance the moduli for small deformations will be directionally dependent, with a strong correlation between the directional dependence and the orientation of the crystalline phase. These seven aspects of the modeling process are represented schematically in Fig.2. In order to model the behavior of these materials undergoing complex deformations at different temperatures, it is important to have a fully invariant three-dimensional model in place that can include these different effects.

In this paper, we model the shape memory behavior in crystallizable triple shape memory polymers in a mechanical setting, with the understanding that this is the first step in developing a full thermodynamic model for these materials. The aim of this paper is to clarify the mechanical issues relating to the triple shape memory polymers. To do so we note that for materials such as TSMPs where in the temporary shapes the material is a mixture of different phases, their behavior cannot be described by traditional theories based on single natural configuration (usually taken to be a stress free state). For this reason the model for crystallization used in this work has been developed within the frame-work of multiple natural configurations. This approach has been used to explain the material response of a large class of materials in a unifying frame-work: multi-network theory [17], twinning [19], traditional plasticity [21], solid to solid phase transitions [22] and viscoelastic liquids [23] have all been modeled within this frame-work, and classical elasticity and classical linearly viscous fluids arise naturally as sub-cases. Furthermore, we have polymer crystallization extensively, particularly the transition from a melt to a semi-crystalline solid using this approach with good success (see [24, 25, 26, 27, 30, 31]). Thermo-mechanical behavior of dual shape memory polymers has also been explained using this approach [43, 44]. Light activated SMPs or LASMPs have also been successfully modeled using this approach [49].

In this paper, we present constitutive equations for the amorphous polymer and the different semicrystalline polymer domains. We also look carefully at the implications of the natural configurations associated with the different crystalline domains on the different temporary shapes occupied by the polymer; and return of the polymer from its second temporary shape to its first temporary shape and then to its original shape. Specifically, we show that the model for the TSMPs that arises using the assumption that the different polymer domains crystallize in a stress-free state, is able to capture the experimental observations accurately. To illustrate the efficacy of the model developed, we solve a typical uni-axial cycle of deformation, we look at crystallization under constant strain and crystallization under constant stress. In addition we solve the uni-axial cycle of deformation for different Triple Shape Creation Procedures (TSCPs). 


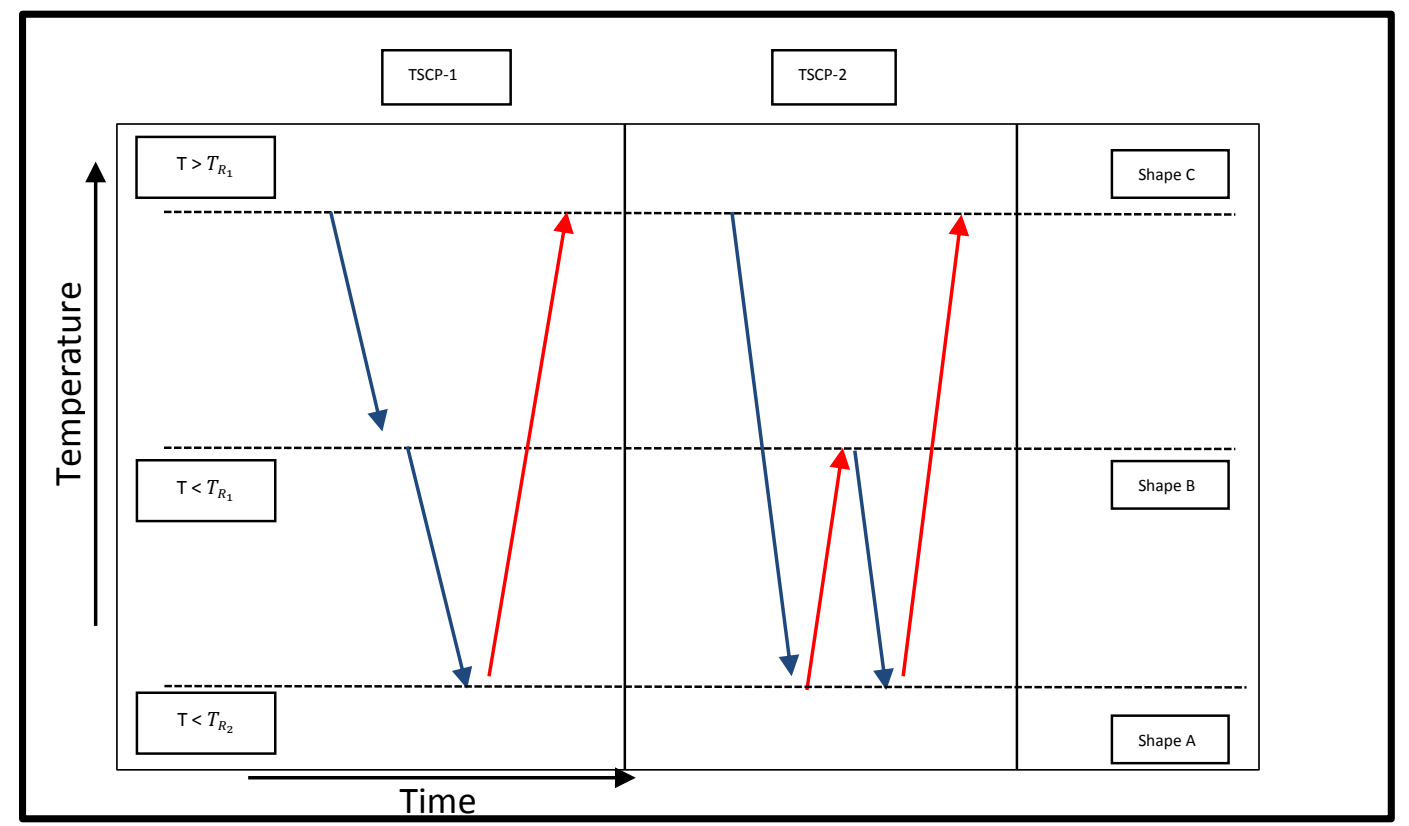

Figure. 3 The temperature (and hence crystallization) history used for two different types of tripe shape creation processes (TSCP-1 and TSCP-2).

\subsection{Triple Shape Creation Procedures (TSCPs)}

The shape changes in TSMPs correspond to the recovery of deformations in reverse order, which were programmed or fixed at elevated temperature levels by application of external stress [42, 45, 46]. Triple shape creation procedure (TSCP) is a process of programing the TSMP in order to create a desired triple shape effect. The triple shape effect displayed by the polymer networks can be influenced by varying the programming procedure followed in a TSCP through variation of the sequence of programming temperatures (see [40], [41], [42]). Furthermore, the application of different TSCPs enables to a certain extent the control of triple-shape capability by influencing the crystallization behavior of the different crystalline phases in these polymers (for details see [41], [42]). Here, we study four different types of TSCPs that have been reported.

\subsubsection{TSCP-1}

This triple shape programming procedure comprises of two steps which are applied to create the two temporary shapes of the polymer as can been seen in Fig.3. The shape changes in the polymer are due to the recovery of two different deformations in a reverse order, which were fixed previously at higher temperature levels $[41,42,45,46]$. TSCP-1 starts from the permanent shape (C), the first deformation is applied at $T_{H}$ which is above the transition temperatures associated with both the switching domains or phases $\left(T_{L}<T_{R_{2}}<T_{\text {mid }}<T_{R_{1}}<T_{H}\right)$. Subsequently, the external stress or strain is maintained and polymer is cooled to a temperature $T_{\text {mid }}$ which is between $T_{R_{1}}$ and $T_{R_{2}}$. On cooling below the first recovery temperature $T_{R_{1}}$, the first switching domain or phase-1 crystallizes, these newly formed crystallites act as physical crosslinks and fix the first temporary shape (B) upon unloading. At $T_{\text {mid }}$ a 
successive deformation is applied and the polymer is subsequently cooled to a temperature $T_{L}$, which is below both the transition temperatures. At this point second switching domain or phase- 2 crystallizes along with a portion of phase- 1 that did not crystallize at the higher temperature, this results in fixation of second temporary shape (A) upon unloading. Following this as the polymer is heated above the second recovery temperature $T_{R_{2}}$ crystallites of phase- 2 melt and polymer retracts towards first temporary shape (B). As phase-1 that crystallized at the lower temperature is still present the first temporary shape (B) is not recovered fully, this is a drawback of TSCP-1 (see [42]). On subsequent heating above the first recovery temperature $T_{R_{1}}$, phase-1 that crsytallized at both the higher and lower temperatures melts and the original shape is recovered as the crystallites that act as physical crosslinks are no longer available to maintain the temporary shape. The different stages of the cycle are shown in Fig. 3.

\subsubsection{TSCP-2}

A limitation of TSCP-1 is that a portion of phase-1 crystallizes at the lower temperature along with phase2 and contributes to the fixation of second temporary shape. As the polymer is heated above the second recovery temperature $T_{R_{2}}$, phase-2 melts and the polymer retracts towards the first temporary shape. However, as the portion of phase-1 that crystallized at a lower temperature does not melt on heating above $T_{R_{2}}$ the first temporary shape is not recovered fully after heating; this negatively affects the fixation of shape during the first programming/cooling step. TSCP-2 is a slight modification of TSCP-1; Fig.3 shows the difference in temperature history between TSCP-1 and TSCP-2. By application of TSCP-2 the crystallization of phases or switching domains could be influenced by varying the sequence of programming temperatures which leads to enhanced triple shape characteristics of the polymer (see [42]).

In TSCP-2, the polymer is deformed at the higher temperature $T_{H}$ and the first cooling step after the deformation is continued, till a temperature $T_{L}$ which is below both the recovery temperatures is reached $\left(T_{L}<T_{R_{2}}<T_{\text {mid }}<T_{R_{1}}<T_{H}\right)$. This leads to crystallization of phase-2 and a complete crystallization of phase- 1 both at the higher and lower transition temperatures. After this the material is unloaded. The polymer is subsequently heated to a temperature $T_{\text {mid }}$, which is above the second recovery temperature, this results in melting of the phase- 2 crystalline domains. After this melting process, the first temporary shape is obtained which is fixed completely by crystallites of phase-1 Subsequently, the polymer is deformed further and cooled below the second recovery temperature to a temperature $T_{L}$, at this point phase- 2 crystallizes again, this fixes the second temporary shape upon unloading. As Phase- 1 has already crystallized completely during the first cooling process, it does not crystallize during this second crystallization process and hence does not contribute to the fixation of the second temporary shape. In this way, using TSCP-2 the fixation of the two temporary shapes is limited to only one phase respectively. On subsequent heating above the second recovery temperature $T_{R_{2}}$, phase- 2 melts and first temporary shape is recovered. On further heating above the first recovery temperature $T_{R_{1}}$, phase-1 melts and the polymer reverts back to the permanent shape. TSCP-2 thus provides a better control over the triple shape effect [42]. 


\subsubsection{TSCP-3}

In this procedure the TSMPs exhibit a triple-shape behavior under constant stress, which is based upon elongation caused by crystallization and contraction caused by melting. Here, a TSMP which is subjected to a constant external stress is able to reversibly switch between the permanent shape and two temporary shapes, when it is repeatedly stimulated only by cyclic temperature changes between $T_{H}$ and $T_{L}$ (see [40], [41]). The triple shape effect using TSCP-1 and TSCP-2 is a one way effect as another programming process needs to be applied after the complete shape recovery of the permanent shape for enabling a new triple shape memory effect. TSCP-3 starts from the permanent shape (C) and the first deformation is applied at $T_{H}$. Subsequently, the external stress is maintained and polymer is cooled to a temperature $T_{\text {mid }}$ below the first recovery temperature, phase- 1 crystallizes at this point. Because the newly formed crystalline material is formed in a stress free state, while the stress on the polymer is kept constant, the polymer will stretch as the crystallization proceeds, this will be explained in more detail in the next section. This elongation during crystallization is responsible for fixation of the first temporary shape (B). On further cooling to temperature $T_{L}$ which is below both the transition temperatures phase- 2 crystallizes along with a portion of phase- 1 that did not crystallize at the higher temperature. Again, as the newly formed crystalline material is formed in a stress free state, while the stress on the polymer is kept constant, the polymer will stretch as the crystallization proceeds. This crystallization induced elongation fixes the second temporary shape (A). Subsequently, as the polymer is heated above the second recovery temperature $T_{R_{2}}$ crystalline phase-2 melts and the polymer retracts towards the first temporary shape (B). The shape (B) fixed during cooling differs slightly from the shape (B) recovered during heating. This is because the phase-1 formed at lower temperature also contributes to the fixation of shape (A) but does not melt at this point [40]. On further heating above the first recovery temperature $T_{R_{1}}$, phase-1 crystallized both at the higher and lower temperature melts and the polymer retracts to its original shape. TSCP-3 is thus a reversible process as the changes in shape can be triggered by cyclic changes in temperature and another programming process is not required after the shape recovery to enable a new triple shape effect. The reversibility of the triple shape effect can be used for creation of actuators that have the ability to perform complex shape changes repeatedly, which are solely controlled by temperature while they are being held under constant stress (see [40, 41]).

\subsubsection{TSCP-4}

This is a one-step triple shape creation programming process, where the polymer is deformed only once at a temperature $T_{H}$ above both the recovery temperatures and then cooled to below $T_{L}$ while keeping the stress constant. Once it has been cooled below $T_{L}$ the polymer is unloaded. Now on heating above the two recovery temperatures, two distinct thermally induced shape changes are obtained sequentially (see [41]). As only one deformation is need be applied during the programming process this is a simple way to create a triple shape effect. In TSCP-4, the polymer is deformed at a temperature $T_{H}$ which is above the recovery temperatures of both the phases and cooled to a temperature $T_{L}$ which, is below both the recovery temperatures $\left(T_{L}<T_{R_{2}}<T_{\text {mid }}<T_{R_{1}}<T_{H}\right)$ while keeping the stress constant. During this cooling process both phase- 1 and phase- 2 crystallize and upon unloading the first temporary shape is fixed. On 
subsequent heating above the second recovery temperature $T_{R_{2}}$ phase-2 melts and the polymer reverts to a second temporary shape, as it cannot revert to the permanent shape due to the presence of the crystallites of phase-1. On further heating above the first recovery temperature $T_{R_{1}}$ crystallites of phase- 1 melt and the polymer reverts back to its permanent shape. Thus, a triple shape effect can be observed during the recovery process upon heating.

In this paper we examine these four TSCP and it should be noted that other shape creation processes are possible. This is another example of the versatility of TSMP's, which should help them in finding uses in different applications.

\section{Preliminaries}

Consider a body $B$ in the reference configuration $\kappa_{0}$. Let $\mathbf{X}$ denote a typical position of a material point in $\kappa_{0}$. Let $\kappa_{t}$ be the configuration of the body at a time t. Then the motion $\chi_{\kappa_{0}}$ assigns to each particle in configuration $\kappa_{0}$ a position in the configuration $\kappa_{t}$ at time t, i.e.

$$
\mathbf{x}=\chi_{\kappa_{0}}(\mathbf{X}, t)
$$

The deformation gradient $\mathbf{F}_{\kappa_{R}}$ is defined through

$$
\mathbf{F}_{\kappa_{R}}=\frac{\partial \chi_{\kappa_{0}}}{\partial \mathbf{X}}
$$

The left and right Cauchy-Green tensors are defined through

$$
\begin{aligned}
& \mathbf{B}_{\kappa_{0}}=\mathbf{F}_{\kappa_{0}} \mathbf{F}^{\mathrm{T}}{ }_{\kappa_{0}} \\
& \mathbf{C}_{\kappa_{0}}=\mathbf{F}_{\kappa_{0}}^{\mathrm{T}} \mathbf{F}_{\kappa_{0}}
\end{aligned}
$$

Any acceptable process has to satisfy the appropriate balance laws. The balance equations appropriate for studying a process in a mechanical setting are the conservation of mass, linear and angular momentum. We assume that the material is incompressible and the conservation of mass reduces to

$$
\operatorname{div}(\mathbf{v})=0
$$

where, $\mathbf{v}$ is the velocity. The conservation of linear momentum is given by

$$
\rho\left[\frac{\partial \mathbf{v}}{\partial t}+[\nabla \mathbf{v}] \mathbf{v}\right]=\operatorname{div} \mathbf{T}^{\mathrm{T}}+\rho \mathbf{g}
$$

where, $\mathbf{g}$ is the acceleration due to gravity, $\rho$ is the density, $\nabla \mathbf{v}$ is the Eulerian velocity gradient and is sometimes denoted by $\mathbf{L}$ and $\mathbf{T}$ is the Cauchy stress tensor. For an incompressible material, the stress tensor $\mathbf{T}$ reduces to 


$$
\mathbf{T}=-p \mathbf{I}+\mathbf{T}^{E}
$$

where, $p$ is the indeterminate part of the stress due to the constraint of incompressibility, and $\mathrm{T}^{E}$ is the constitutively determined extra stress. The balance of angular momentum for a body requires that the stress tensor be symmetric.

\section{Constitutive model}

The modeling of TSMPs can be typically outlined into eight parts, namely the rubbery phase (at temperature above both the recovery temperatures $T_{R_{1}}$ and $T_{R_{2}}$ ), the first semi-crystalline phase (below the first recovery temperature $T_{R_{1}}$ ), the semi-crystalline phase with a mixture of two crystalline phases (below the second recovery temperature $T_{R_{2}}$ ), the first crystallization process (on cooling below the first recovery temperature $T_{R_{1}}$ ), the second crystallization process (on cooling below the second recovery temperature $T_{R_{2}}$ ), the first melting process (on heating back above the second recovery temperature $T_{R_{2}}$ ) and the second melting process (on heating back above the first recovery temperature $T_{R_{1}}$ ). Here each of these parts is addressed separately.

\subsection{Rubbery Amorphous Phase}

Above both the recovery temperatures the material behaves like an elastomer. Polymers exhibiting rubber like elasticity can be modeled using a variety of constitutive equations that are available in literature, such as the Neo-Hookean model, the Mooney Rivilin model etc. Elastomers are usually modeled as incompressible hyperelastic materials. The Cauchy stress $\mathbf{T}$ for an incompressible hyperelastic material is given by:

$$
\mathbf{T}=-p \mathbf{I}+2 \rho \mathbf{F}_{\kappa_{0}} \frac{\partial \psi_{a}}{\partial \boldsymbol{C}_{\kappa_{0}}} \mathbf{F}_{\kappa_{0}}^{T}
$$

where, $\mathrm{p}$ is the Lagrange multiplier due to the constraint of incompressibility, $\mathbf{F}_{\kappa_{0}}$ is the deformation gradient measured from some fixed configuration $\kappa_{a}$ associated with the amorphous rubbery phase, $\psi_{a}$ is the Helmholtz potential or the stored energy. Details on the constitutive equations for rubber like materials can be found in Treolar [37] and Ogden [15].

The elastic behavior in rubber like materials is mainly due to changes in conformational entropy due to deformation. Specific models to describe the behavior of rubbery phase of the SMP can be chosen by picking forms for the stored energy that are consistent with experimental results. Here, it should be noted that most elastomers exhibit viscoelastic behavior, this can be easily incorporated into the model, and however, for the sake of clarity we do not discuss this issue here. Note, the material used to obtain the experimental data (see [40]) that were used for comparison with the model, do not exhibit any significant viscoelastic behavior. The material is held at constant stress for extended periods [40] of time without any 
evidence of creep. Change in length takes place near the melting and crystallization temperatures and is associated with phase change. Hence the models developed in this paper are appropriate for these type of materials.

\subsection{Crystallization Rate}

In a full thermodynamic framework an activation criterion that depends on various thermodynamic variables would have to be prescribed. The polymer begins to crystallize after the activation criterion is met and the rate of crystallization comes out of the thermodynamics associated with the problem (see Rao and Rajagopal [27]) [44]. In this paper, however, we directly prescribe a crystallization rate equation, as the main purpose of this paper is to understand the mechanical behavior of the TSMP.

\subsection{Rigid semi-crystalline Phases:}

Crystallization generally takes place in a gradual manner and does not occur all at an instant. Constitutive assumptions have to be made for this mixed region in order to model the material. This mixture of the crystalline phases formed both at the higher and the lower temperatures and amorphous phases is treated as a constrained mixture. We allow co-occupancy of the phases in an averaged sense as is done in traditional mixture theory (see $[1,2,38,20]$ ). In addition, it is assumed that the amorphous and crystalline components are constrained to move together, which for polymers is a reasonable assumption as the same molecule traverses both the amorphous and crystalline phases and the presence of cross-links and crystallites prevents the diffusion of individual polymer molecules. The newly formed crystalline materials at the higher and lower temperatures are assumed to be elastic solids. For details (see [43], [29], [12], [13]). For details on how anisotropy can be induced in the crystalline phase (see [25, 26]). We assume that the stored energy of the different phases present are additive, if crystallization takes place with the strain held constant as shown in Fig.2 , then the Helmholtz potential of the mixture is simply given by:

$$
\psi=\alpha_{1_{H}} \psi_{C_{1_{H}}}+\alpha_{1_{L}} \psi_{C_{1_{L}}}+\alpha_{2} \psi_{C_{2}}+\left(1-\alpha_{1_{H}}-\alpha_{1_{L}}-\alpha_{2}\right) \psi_{a}
$$

where, $\psi_{C_{1_{H}}}, \psi_{C_{1_{L}}}$ and $\psi_{C_{2}}$ are the Helmholtz potentials associated with the first crystalline phase formed at the higher and the lower temperatures and the second crystalline phase respectively, $\alpha_{1_{H}}$ and $\alpha_{1_{L}}$ are the amounts of crystallinity of first crystalline phase formed at the higher and lower temperatures respectively, and $\alpha_{2}$ is the amount of crystallinity of the second crystalline phase. Note, the first crystalline phase begins to crystallize below $T_{R_{1}}$ and continues to crystallize below $T_{R_{2}}$. We have split the amount crystallized into two parts, $\alpha_{1_{H}}$ represents the amount crystallized in the first temperature interval $T_{\text {mid }}<T_{R_{1}}<T_{H}$. While, $\alpha_{1_{L}}$ represents the first crystalline phase that crystallizes in the second temperature interval $T_{L}<T_{R_{2}}<T_{\text {mid }}$. Also we allow for different stored energies for these two phases of crystallization, $\psi_{C_{1_{H}}}$ and $\psi_{C_{2}}$. For such case the stress in the mixture can be shown to be given by: 


$$
\begin{aligned}
& \mathbf{T}=-\mathrm{p} \mathbf{I}+\left(1-\alpha_{1_{H}}-\alpha_{1_{L}}-\alpha_{2}\right) 2 \rho \mathbf{F}_{\kappa_{a}} \frac{\partial \psi_{a}}{\partial \boldsymbol{C}_{\kappa_{a}}} \mathbf{F}_{\kappa_{a}}^{T}+\alpha_{1_{H}} 2 \rho \mathbf{F}_{\kappa_{c_{1} H}} \frac{\partial \psi_{c_{1_{H}}}}{\partial \mathbf{C}_{\kappa_{c_{1_{H}}}}} \mathbf{F}_{\kappa_{c_{1_{H}}}}^{T} \\
& +\alpha_{1_{L}} 2 \rho \mathbf{F}_{\kappa_{c_{1_{L}}}} \frac{\partial \psi_{c_{1_{L}}}}{\partial \mathbf{C}_{\kappa_{c_{1_{L}}}}} \mathbf{F}_{\kappa_{c_{1_{L}}}}^{T}+\alpha_{2} 2 \rho \mathbf{F}_{\kappa_{c_{2}}} \frac{\partial \psi_{c_{2}}}{\partial \mathbf{C}_{\kappa_{c_{2}}}} \mathbf{F}_{\kappa_{c_{2}}}^{T} \\
& =-\mathrm{p} \mathbf{I}+\left(1-\alpha_{1_{H}}-\alpha_{1_{L}}-\alpha_{2}\right) \widehat{\mathbf{T}}_{a}+\alpha_{1_{H}} \widehat{\mathbf{T}}_{c_{1_{H}}}+\alpha_{1_{L}} \widehat{\mathbf{T}}_{c_{1_{L}}}+\alpha_{2} \widehat{\mathbf{T}}_{c_{2}}
\end{aligned}
$$

where, $\kappa_{c_{1_{H}}}, \kappa_{c_{1_{L}}}$ and $\kappa_{c_{2}}$ are natural configurations associated with the first phase crystallized at higher and lower temperatures and the second crystalline phase respectively. Equation shows that for the limiting case of crystalline phase formed in the same state at all times, a rule of mixtures approximation is recovered, where $\widehat{\mathbf{T}}_{a}, \widehat{\mathbf{T}}_{c_{1 H}}, \widehat{\mathbf{T}}_{c_{1 L}}$ and $\widehat{\mathbf{T}}_{c_{2}}$ can be interpreted as the stresses present in pure amorphous phase and the pure crystalline phases respectively. If however, the crystallization takes place while the TSMP is being deformed, the crystalline phase is not all formed in the same configuration, consequently the stored energy of the different crystalline phases $\psi_{c_{1_{H}}}, \psi_{c_{1_{L}}}$ and $\psi_{c_{2}}$ changes with time, to account for this Eq.(9) is replaced by the following equation:

$$
\begin{aligned}
& \psi=\left(1-\alpha_{1_{H}}-\alpha_{1_{L}}-\alpha_{2}\right) \psi_{a}+\left[\int_{t_{1}}^{t_{2}} \psi_{C_{1_{H}}(\tau)} \frac{d \alpha_{1_{H}}}{d \tau} d \tau\right]_{H} \\
& +\left[\int_{t_{3}}^{t_{4}} \psi_{C_{1_{L}}(\tau)} \frac{d \alpha_{1_{L}}}{d \tau} d \tau+\int_{t_{3}}^{t_{4}} \psi_{C_{2}(\tau)} \frac{d \alpha_{2}}{d \tau} d \tau\right]_{L}
\end{aligned}
$$

The integrals are introduced to capture the variation in the Helmholtz potentials associated with the different crystalline phases $\psi_{C_{1_{H}(\tau)}}, \psi_{C_{L_{L}(\tau)}}$ and $\psi_{C_{2(\tau)}}$ with time. Here, $t_{1}$ is the time at which crystallization of the first phase at higher temperature is initiated, $t_{2}$ is the time at which it is terminated, $t_{3}$ is the time at which crystallization at the lower temperature is initiated, $t_{4}$ is the time at which it is terminated. $\tau$ is some intermediate time between times $t_{1}$ and $t_{2}$ and between times $t_{3}$ and $t_{4}$. Also, the functional form for the Helmholtz potentials for the different fractions of the different crystalline phases formed at different times can in general be different depending on the local conditions. In addition the invariance associated with these different fractions can be different, chosen in a way to reflect the symmetry associated with the crystalline phase. The equations for the stress can be shown to reduce to (for a detailed derivation see Rao and Rajagopal [24, 26]):

$$
\begin{aligned}
& \mathbf{T}=-\mathrm{p} \mathbf{I}+\left(1-\alpha_{1_{H}}-\alpha_{1_{L}}-\alpha_{2}\right) 2 \rho \mathbf{F}_{\kappa_{a}} \frac{\partial \psi_{a}}{\partial \mathbf{C}_{\kappa_{a}}} \mathbf{F}_{\kappa_{a}}^{T}+\int_{t_{1}}^{t_{2}} 2 \rho \mathbf{F}_{\kappa_{c_{1_{H}}(\tau)}} \frac{\partial \psi_{c_{1_{H}(\tau)}}}{\partial \mathbf{C}_{\kappa_{c_{1_{H}}(\tau)}}} \mathbf{F}_{\kappa_{c_{1_{H}}(\tau)}} \frac{d \alpha_{1_{H}}}{d \tau} d \tau \\
& +\int_{t_{3}}^{t_{4}} 2 \rho \mathbf{F}_{\kappa_{c_{L_{L}}(\tau)}} \frac{\partial \psi_{c_{1_{L}(\tau)}}}{\partial \mathbf{C}_{\kappa_{c_{1_{L}(\tau)}}}} \mathbf{F}_{\kappa_{c_{L_{L}}(\tau)}}^{T} \frac{d \alpha_{1_{L}}}{d \tau} d \tau+\int_{t_{3}}^{t_{4}} 2 \rho \mathbf{F}_{\kappa_{c_{2}(\tau)}} \frac{\partial \psi_{c_{\kappa_{c_{2}(\tau)}}}}{\partial \mathbf{C}_{\kappa_{\kappa_{c_{2}(\tau)}}}} \mathbf{F}_{\kappa_{\kappa_{c_{2}(\tau)}}}^{T} \frac{d \alpha_{2}}{d \tau} d \tau
\end{aligned}
$$


where, $\kappa_{C_{I_{H}(\tau)}}$, is the stress free state associated with the first crystalline phase formed at a higher temperature at some intermediate time $\tau$ between times $t_{1}$ and $t_{2}, \kappa_{C_{L_{L}(\tau)}}$ is the stress free state associated with the first crystalline phase formed at a lower temperature at some intermediate time $\tau$ between times $t_{3}$ and $t_{4}, \kappa_{C_{2(\tau)}}$ is the stress free state associated with the second crystalline phase formed at a lower



$$
t_{1}<\tau_{1}<t_{2}<t_{3}<\tau_{2}<t_{4}<t
$$

Figure 4. Natural configurations associated with the unloading process after the crystallization processes at both the higher and lower temperatures are complete.

temperature at some intermediate time $\tau$ between times $t_{3}$ and $t_{4}$. If the newly formed crystalline phases formed at each different instances are orthotropic (see Rao and Rajagopal [24, 26]) the stress simplifies to: 


$$
\begin{aligned}
& \mathbf{T}=-\mathrm{p} \mathbf{I}+\left(1-\alpha_{1_{H}}(t)-\alpha_{1_{L}}(t)-\alpha_{2}(t)\right) \mu_{a} \mathbf{B}_{\kappa_{a}}+2 \rho \int_{t_{1}}^{t_{2}} c_{1} \mathbf{B}_{\kappa_{c_{l_{H}}(\tau)}} \frac{d \alpha_{1_{H}}}{d \tau} d \tau \\
& +4 \rho \int_{t_{1}}^{t_{2}}\left(\mathbf{F}_{\kappa_{c_{I_{H}}(\tau)}}\left(c_{2}\left(J_{1}-1\right) \mathbf{n}_{\kappa_{c_{c_{H}}(\tau)}} \otimes \mathbf{n}_{\kappa_{c_{c_{H}}(\tau)}}+c_{3}\left(K_{1}-1\right) \mathbf{m}_{\kappa_{c_{c_{H}}(\tau)}} \otimes \mathbf{m}_{\kappa_{c_{c_{H}}(\tau)}}\right) \mathbf{F}_{\kappa_{c_{c_{H}}(\tau)}}^{T}\right) \\
& \frac{d \alpha_{1_{H}}}{d \tau} d \tau+2 \rho \int_{t_{3}}^{t_{4}} c_{1}^{\prime} \mathbf{B}_{\kappa_{c_{L}(\tau)}} \frac{d \alpha_{1_{L}}}{d \tau} d \tau \\
& +4 \rho \int_{t_{3}}^{t_{4}}\left(\mathbf{F}_{\kappa_{c_{L_{L}(\tau)}}}\left(c_{2}^{\prime}\left(J_{2}-1\right) \mathbf{n}_{\kappa_{c_{c_{L}(\tau)}}^{\prime}}^{\prime} \otimes \mathbf{n}_{\kappa_{c_{c_{L}(\tau)}}^{\prime}}^{\prime}+c_{3}^{\prime}\left(K_{2}-1\right) \mathbf{m}_{\kappa_{c_{c_{L}(\tau)}}}^{\prime} \otimes \mathbf{m}_{\kappa_{c_{c_{L}(\tau)}}}^{\prime}\right) \mathbf{F}_{\kappa_{c_{c_{L}(\tau)}}}^{T}\right) \\
& \frac{d \alpha_{1_{L}}}{d \tau} d \tau+2 \rho \int_{t_{3}}^{t_{4}} c_{4} \mathbf{B}_{\kappa_{c_{2}(\tau)}} \frac{d \alpha_{2}}{d \tau} d \tau \\
& +4 \rho \int_{t_{3}}^{t_{4}}\left(\mathbf{F}_{\kappa_{c_{2}(\tau)}}\left(c_{5}\left(J_{2}-1\right) \mathbf{n}_{\kappa_{c_{2}(\tau)}} \otimes \mathbf{n}_{\kappa_{c_{2}(\tau)}}+c_{6}\left(K_{2}-1\right) \mathbf{m}_{\kappa_{c_{2}(\tau)}} \otimes \mathbf{m}_{\kappa_{c_{2}(\tau)}}\right) \mathbf{F}_{\kappa_{c_{2}(\tau)}}^{T}\right) \\
& \frac{d \alpha_{2}}{d \tau} d \tau
\end{aligned}
$$

where, $t_{1}$ is the time when crystallization of the first phase is initiated at the higher temperature, $t_{3}$ is the lower temperature when the crystallization is initiated where both the first and the second phase crystallize. $c_{1}, c_{2}, c_{3}$ are material moduli associated with the first phase crystallized at the higher temperature. $c_{4}, c_{5}, c_{6}$ are the material moduli associated with the second phase crystallized at the lower temperature. $c_{1}^{\prime}, c_{2}^{\prime}, c_{3}^{\prime}$ are material moduli associated with the first phase crystallized at the lower temperature. Note, $\mathbf{F}_{\kappa_{c_{1 H}(\tau)}}$ is the mapping between the stress free state of the crystalline phase formed at a time $\tau$ between times $t_{1}$ and $t_{2}$ to the configuration occupied by the polymer at current time $\mathrm{t}, \mathbf{F}_{\kappa_{\varepsilon_{I_{I}}(\tau)}}$ and $\mathbf{F}_{\kappa_{c_{2}(\tau)}}$ are the mapping between the stress free states of the crystalline phases formed at time $\tau$ between times $t_{3}$ and $t_{4}$ to the configuration occupied by the polymer at current time $\mathrm{t}$ (see Fig.4 ). Since the crystalline phase is formed in a stress free state, $\kappa_{c_{l_{H}(\tau)}}$ is the configuration occupied by the polymer at some intermediate time $\tau$ between times $t_{1}$ and $t_{2}$, and $\kappa_{c_{L_{L}(\tau)}}, \kappa_{c_{2(\tau)}}$ are configurations occupied by the polymer at some intermediate time $\tau$ between times $t_{3}$ and $t_{4}$. The unit vectors $\mathbf{n}_{\kappa_{c_{l_{H}}(\tau)}}$ and $\mathbf{m}_{\kappa_{c_{l_{H}(\tau)}}}$ are the directions of orthotropy associated with the first crystalline phase formed at the higher temperature. The unit vectors $\mathbf{n}_{\kappa_{c_{1}(\tau)}}^{\prime}$ and $\mathbf{m}_{\kappa_{c_{c_{L}(\tau)}}^{\prime}}^{\prime}$ are the directions of orthotropy associated with the first crystalline phase formed at the lower temperature. The unit vectors $\mathbf{n}_{\kappa_{c_{2}(\tau)}}$ and $\mathbf{m}_{\kappa_{c_{2}(\tau)}}$ are the directions of orthotropy associated with the second crystalline phase formed at the lower temperature. Details on how they are calculated from the deformation in the amorphous phase can be found in Rao and Rajagopal [24]. The invariants $J_{1}, K_{1}, J_{2}, K_{2}, J^{\prime}{ }_{1}, K^{\prime}{ }_{1}$ are given by 


$$
\begin{aligned}
& \mathbf{J}_{1}=\mathbf{n}_{\kappa_{c_{1_{H}}(\tau)}} \cdot \mathbf{C}_{\kappa_{c_{1_{H}}(\tau)}} \mathbf{n}_{\kappa_{c_{1_{H}}(\tau)}}, \mathbf{K}_{1}=\mathbf{m}_{\kappa_{c_{1_{H}}(\tau)}} \cdot \mathbf{C}_{\kappa_{c_{1_{H}}(\tau)}} \mathbf{m}_{\kappa_{c_{\mathcal{I}_{H}}(\tau)}} \\
& \mathbf{J}_{1}^{\prime}=\mathbf{n}_{\kappa_{c_{1_{L}}(\tau)}^{\prime}}^{\prime} \cdot \mathbf{C}_{\kappa_{c_{1_{L}}(\tau)}} \mathbf{n}_{\kappa_{c_{c_{L}}(\tau)}^{\prime}}^{\prime}, \mathbf{K}_{1}^{\prime}=\mathbf{m}_{\kappa_{c_{1_{L}}(\tau)}}^{\prime} \cdot \mathbf{C}_{\kappa_{c_{1_{L}}(\tau)}} \mathbf{m}_{\kappa_{c_{c_{L}}(\tau)}}^{\prime} \\
& \mathbf{J}_{2}=\mathbf{n}_{\kappa_{c_{2}(\tau)}} \cdot \mathbf{C}_{\kappa_{c_{2}(\tau)}} \mathbf{n}_{\kappa_{c_{2}(\tau)}}, \mathbf{K}_{2}=\mathbf{m}_{\kappa_{c_{2}(\tau)}} \cdot \mathbf{C}_{\kappa_{c_{2}(\tau)}} \mathbf{m}_{\kappa_{c_{2}(\tau)}}
\end{aligned}
$$

In this paper we will use the non-dimensional form of Eq. (12). The non-dimensionalization has been done in the following manner: the stress is non-dimensionalized using the modulus associated with the amorphous phase $\mu_{a}$, this results in the following non-dimensional quantities associated with material moduli related to the crystalline phase, namely,

$\mu_{1}=\frac{2 \rho c_{1}}{\mu_{a}}, \mu_{2}=\frac{4 \rho c_{2}}{\mu_{a}}, \mu_{3}=\frac{4 \rho c_{3}}{\mu_{a}}, \mu_{1}^{\prime}=\frac{2 \rho c_{1}^{\prime}}{\mu_{a}}, \mu_{2}^{\prime}=\frac{4 \rho c_{2}^{\prime}}{\mu_{a}}, \mu_{3}^{\prime}=\frac{4 \rho c_{3}^{\prime}}{\mu_{a}}, \mu_{4}=\frac{2 \rho c_{5}}{\mu_{a}}, \mu_{5}=\frac{4 \rho c_{6}}{\mu_{a}}, \mu_{6}=\frac{4 \rho c_{7}}{\mu_{a}}$.

The dimensionless form of the equation for stress is:

$$
\begin{aligned}
& \mathbf{T}=-\mathrm{p} \mathbf{I}+\left(1-\alpha_{1_{H}}(t)-\alpha_{1_{L}}(t)-\alpha_{2}(t)\right) \mu_{a} \mathbf{B}_{\kappa_{a}}+\int_{t_{1}}^{t_{2}} \mu_{1} \mathbf{B}_{\kappa_{c_{l_{H}}(\tau)}} \frac{d \alpha_{1_{H}}}{d \tau} d \tau
\end{aligned}
$$

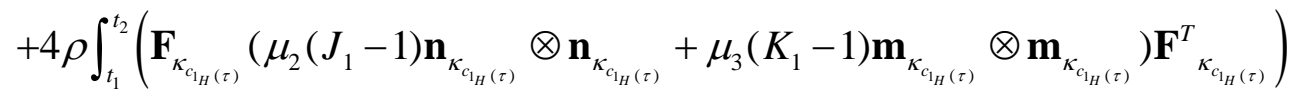

$$
\begin{aligned}
& \frac{d \alpha_{1_{H}}}{d \tau} d \tau+2 \rho \int_{t_{3}}^{t_{4}} \mu_{1}^{\prime} \mathbf{B}_{\kappa_{c_{L}(\tau)}} \frac{d \alpha_{1_{L}}}{d \tau} d \tau \\
& +4 \rho \int_{t_{3}}^{t_{4}}\left(\mathbf{F}_{\kappa_{c_{L_{L}}(\tau)}}\left(\mu_{2}^{\prime}\left(J_{2}-1\right) \mathbf{n}_{\kappa_{c_{L_{L}(\tau)}}^{\prime}}^{\prime} \otimes \mathbf{n}_{\kappa_{c_{c_{L}}(\tau)}^{\prime}}^{\prime}+\mu_{3}^{\prime}\left(K_{2}-1\right) \mathbf{m}_{\kappa_{c_{1_{L}}(\tau)}}^{\prime} \otimes \mathbf{m}_{\kappa_{c_{c_{L}}(\tau)}}^{\prime}\right) \mathbf{F}_{\kappa_{c_{c_{L}}(\tau)}}^{T}\right) \\
& \frac{d \alpha_{1_{L}}}{d \tau} d \tau+2 \rho \int_{t_{3}}^{t_{4}} \mu_{4} \mathbf{B}_{\kappa_{c_{2}(\tau)}} \frac{d \alpha_{2}}{d \tau} d \tau \\
& +4 \rho \int_{t_{3}}^{t_{4}}\left(\mathbf{F}_{\kappa_{c_{2}(\tau)}}\left(\mu_{5}\left(J_{2}-1\right) \mathbf{n}_{\kappa_{c_{2}(\tau)}} \otimes \mathbf{n}_{\kappa_{c_{2}(\tau)}}+\mu_{6}\left(K_{2}-1\right) \mathbf{m}_{\kappa_{c_{2}(\tau)}} \otimes \mathbf{m}_{\kappa_{c_{2}(\tau)}}\right) \mathbf{F}^{T}{ }_{\kappa_{c_{2}(\tau)}}\right) \\
& \frac{d \alpha_{2}}{d \tau} d \tau
\end{aligned}
$$

Finally, we note that if the semi-crystalline solid is subject to inelastic deformations the underlying stress free configurations $\kappa_{a}, \kappa_{c_{1(\tau)}}, \kappa_{c_{L_{L}(\tau)}}$ and $\kappa_{c_{2(\tau)}}$ will evolve and evolution equations will have to be prescribed for these akin to the evolution of plastic strain in plasticity theory.

\subsection{Melting Rate:}

As the polymer is heated above the second recovery temperature $\mathrm{T}_{R_{2}}$ crystallites of the second phase begin to melt and the polymer begins to transform back to the amorphous phase. On subsequent heating above the first recovery temperature $\mathrm{T}_{R_{1}}$ crystallites of the first phase formed both at higher and lower temperatures begin to melt. As more and more of the crystallites melt the behavior of the polymer is increasingly rubber-like, as more of the polymer is released from the crystalline phase. As with the crystallization rates, it is possible to derive the melting rate equations for the different crystalline phases from the thermodynamic considerations, similar to the methodology used to derive the crystallization rate equation in Rao and Rajagopal [27, 30, 31] and Barot et al. [44]. Since for this paper that is not the 
primary thrust, we will prescribe rate equations for the melting of both phases that mimic the general melting behavior. However, it should be noted that these rate equations for melting are an important part of the model as they along with the thermal boundary conditions quantify the time required for the TSMP to transform from the second temporary shape to the first temporary shape and from there to its permanent shape. This is an important facet of the model, which will be explored in the next paper dealing with the full thermodynamic theory.

\section{Application to specific cycle of deformation}

We look at the specific example of a uni-axial cycle of deformation; this is a case of homogeneous deformation. For this typical cycle of deformation four different triple shape creation procedures (TSCPs) have been modeled. The different TSCPs are performed under a set of different thermomechanical programing and recovery conditions. The rates are slow and hence the inertial terms have been ignored.

\subsection{Uni-axial extension}

As, mentioned earlier, for a triple shape memory polymer the study of only the loading process itself is not sufficient. To capture the complete behavior we model the whole cycle, we consider four different triple shape creation procedures. For TSCP-1 the cycle consists of eight distinct phases, loading-1, crystallization-1, unloading-1, loading-2, crystallization-2, unloading-2, melting-1, melting-2. For TSCP2 the cycle consists of nine distinct phases loading-1, crystallization-1, unloading-1, melting-1, loading-2, crystallization-2, unloading-2, melting-2, melting-3. For TSCP-3 the cycle consists of five phases loading-1, crystallization-1, crystallization-2, melting-1, melting-2. For TSCP-4 the cycle consists of six phases loading-1, crystallization-1, unloading-1, crystallization-2, melting-1, melting-2.

We have studied two commonly encountered cases of crystallization, the first involves crystallization under constant strain. The polymer is stretched to a prescribed length, and while the length is kept constant crystallization is initiated by cooling. In the second case, the behavior of the polymer is studied when the crystallization is initiated while keeping the stress constant.

Uni-axial extension for an incompressible material is given by:

$$
x=\Lambda(t) X, \quad y=\frac{1}{\sqrt{\Lambda(t)}} Y, z=\frac{1}{\sqrt{\Lambda(t)}} Z
$$

where, $\mathrm{X}, \mathrm{Y}, \mathrm{Z}$ are the co-ordinates in the undeformed configuration and $\mathrm{x}, \mathrm{y}, \mathrm{z}$ are the co-ordinates in the deformed configuration and $\Lambda(t)$ is the stretch ratio. Here the polymer is extended in the $\mathrm{x}$-direction. For such a motion the tensors $\mathbf{F}_{\kappa_{a}}, \mathbf{F}_{\kappa_{c_{1_{H}}(\tau)}}, \mathbf{F}_{\kappa_{c_{1_{L}}(\tau)}}$ and $\mathbf{F}_{\kappa_{c_{2}(\tau)}}$ are given by:

$$
\mathbf{F}_{\kappa_{a}}=\operatorname{diag}\left(\Lambda(t), \sqrt{\frac{1}{\Lambda(t)}}, \sqrt{\frac{1}{\Lambda(t)}}\right), \mathbf{F}_{\kappa_{c_{H_{H}}(\tau)}}=\operatorname{diag}\left(\frac{\Lambda(t)}{\Lambda(\tau)}, \sqrt{\frac{\Lambda(\tau)}{\Lambda(t)}}, \sqrt{\frac{\Lambda(\tau)}{\Lambda(t)}}\right)
$$




$$
\mathbf{F}_{\kappa_{c_{L_{L}(\tau)}}}=\operatorname{diag}\left(\frac{\Lambda(t)}{\Lambda(\tau)}, \sqrt{\frac{\Lambda(\tau)}{\Lambda(t)}}, \sqrt{\frac{\Lambda(\tau)}{\Lambda(t)}}\right), \mathbf{F}_{\kappa_{c_{2}(\tau)}}=\operatorname{diag}\left(\frac{\Lambda(t)}{\Lambda(\tau)}, \sqrt{\frac{\Lambda(\tau)}{\Lambda(t)}}, \sqrt{\frac{\Lambda(\tau)}{\Lambda(t)}}\right)
$$

Other kinematical tensors namely $\mathbf{B}_{\kappa_{a}}, \mathbf{B}_{\kappa_{c_{1 H}(\tau)}}, \mathbf{B}_{\kappa_{c_{c_{L}}(\tau)}}$ and $\mathbf{B}_{\kappa_{c_{2}(\tau)}}$ and $\mathbf{C}_{\kappa_{a}}, \mathbf{C}_{\kappa_{\kappa_{1_{H}}(\tau)}}, \mathbf{C}_{\kappa_{c_{L_{L}}(\tau)}}$ and $\mathbf{C}_{\kappa_{c_{2}(\tau)}}$ are also diagonal and are given by:

$$
\begin{gathered}
\mathbf{B}_{\kappa_{a}}=\mathbf{C}_{\kappa_{a}}=\operatorname{diag}\left(\Lambda(t)^{2}, \sqrt{\frac{1}{\Lambda(t)}}, \sqrt{\frac{1}{\Lambda(t)}}\right) \\
\mathbf{B}_{\kappa_{c_{I_{H}(\tau)}}}=\mathbf{C}_{\kappa_{c_{I_{H}(\tau)}}}=\operatorname{diag}\left(\left(\frac{\Lambda(t)}{\Lambda(\tau)}\right)^{2}, \sqrt{\frac{\Lambda(\tau)}{\Lambda(t)}}, \sqrt{\frac{\Lambda(\tau)}{\Lambda(t)}}\right) \\
\mathbf{B}_{\kappa_{c_{c_{L}(\tau)}}}=\mathbf{C}_{\kappa_{c_{L_{L}(\tau)}}}=\operatorname{diag}\left(\left(\frac{\Lambda(t)}{\Lambda(\tau)}\right)^{2}, \sqrt{\frac{\Lambda(\tau)}{\Lambda(t)}}, \sqrt{\frac{\Lambda(\tau)}{\Lambda(t)}}\right) \\
\mathbf{B}_{\kappa_{c_{2}(\tau)}}=\mathbf{C}_{\kappa_{c_{2}(\tau)}}=\operatorname{diag}\left(\left(\frac{\Lambda(t)}{\Lambda(\tau)}\right)^{2}, \sqrt{\frac{\Lambda(\tau)}{\Lambda(t)}}, \sqrt{\frac{\Lambda(\tau)}{\Lambda(t)}}\right)
\end{gathered}
$$

For uni-axial extension, as the polymer is stretched in one primary direction, the resulting crystalline phase is transversely isotropic. The resulting stress tensor is identical to the form used in Rao and Rajagopal [24]. Using Eq. (19), Eq.(20), Eq.(21) and Eq.(14), the stress tensor for uni-axial extension is given by: 


$$
\begin{aligned}
& \mathbf{T}=-\mathrm{p} \mathbf{I}+\left(1-\alpha_{1_{H}}(t)-\alpha_{1_{L}}(t)-\alpha_{2}(t)\right) \mathbf{B}_{\kappa_{a}}+\mu_{1} \int_{t_{1}}^{t_{2}} \operatorname{diag}\left(\left(\frac{\Lambda(t)}{\Lambda(\tau)}\right)^{2}, \frac{\Lambda(\tau)}{\Lambda(t)}, \frac{\Lambda(\tau)}{\Lambda(t)}\right) \frac{d \alpha_{1_{H}}}{d \tau} d \tau \\
& +\mu_{2} \int_{t_{1}}^{t_{2}} \operatorname{diag}\left(\left(\left(\frac{\Lambda(t)}{\Lambda(\tau)}\right)^{2}-1\right)\left(\frac{\Lambda(t)}{\Lambda(\tau)}\right)^{2}, 0,0\right) \frac{d \alpha_{1_{H}}}{d \tau} d \tau \\
& +\mu_{1}^{\prime} \int_{t_{3}}^{t_{4}} \operatorname{diag}\left(\left(\frac{\Lambda(t)}{\Lambda(\tau)}\right)^{2}, \frac{\Lambda(\tau)}{\Lambda(t)}, \frac{\Lambda(\tau)}{\Lambda(t)}\right) \frac{d \alpha_{1_{L}}}{d \tau} d \tau \\
& +\mu_{2}^{\prime} \int_{t_{3}}^{t_{4}} \operatorname{diag}\left(\left(\left(\frac{\Lambda(t)}{\Lambda(\tau)}\right)^{2}-1\right)\left(\frac{\Lambda(t)}{\Lambda(\tau)}\right)^{2}, 0,0\right) \frac{d \alpha_{1_{L}}}{d \tau} d \tau \\
& +\mu_{4} \int_{t_{3}}^{t_{4}} \operatorname{diag}\left(\left(\frac{\Lambda(t)}{\Lambda(\tau)}\right)^{2}, \frac{\Lambda(\tau)}{\Lambda(t)}, \frac{\Lambda(\tau)}{\Lambda(t)}\right) \frac{d \alpha_{2}}{d \tau} d \tau \\
& +\mu_{5} \int_{t_{3}}^{t_{4}} \operatorname{diag}\left(\left(\left(\frac{\Lambda(t)}{\Lambda(\tau)}\right)^{2}-1\right)\left(\frac{\Lambda(t)}{\Lambda(\tau)}\right)^{2}, 0,0\right) \frac{d \alpha_{2}}{d \tau} d \tau
\end{aligned}
$$

This equation will be simplified for the different stages of the triple shape memory cycle. For a uni-axial extension, if the lateral surfaces are stress free, the stress in the direction of extension is given by:

$$
\begin{aligned}
& \mathrm{T}_{11}=\left(1-\alpha_{1_{H}}(t)-\alpha_{1_{L}}(t)-\alpha_{2}(t)\right)\left(\Lambda(t)^{2}-\frac{1}{\Lambda(t)}\right)+\mu_{1} \int_{t_{1}}^{t_{2}}\left(\left(\frac{\Lambda(t)}{\Lambda(\tau)}\right)^{2}-\frac{\Lambda(\tau)}{\Lambda(t)}\right) \frac{d \alpha_{1_{H}}}{d \tau} d \tau \\
& \mu_{2} \int_{t_{1}}^{t_{2}}\left(\left(\left(\frac{\Lambda(t)}{\Lambda(\tau)}\right)^{2}-1\right)\left(\frac{\Lambda(t)}{\Lambda(\tau)}\right)^{2}\right) \frac{d \alpha_{1_{H}}}{d \tau} d \tau+\mu_{1}^{\prime} \int_{t_{3}}^{t_{4}}\left(\left(\frac{\Lambda(t)}{\Lambda(\tau)}\right)^{2}-\frac{\Lambda(\tau)}{\Lambda(t)}\right) \frac{d \alpha_{1_{L}}}{d \tau} d \tau \\
& +\mu_{2}^{\prime} \int_{t_{3}}^{t_{4}}\left(\left(\left(\frac{\Lambda(t)}{\Lambda(\tau)}\right)^{2}-1\right)\left(\frac{\Lambda(t)}{\Lambda(\tau)}\right)^{2}\right) \frac{d \alpha_{1_{L}}}{d \tau} d \tau+\mu_{4} \int_{t_{3}}^{t_{4}}\left(\left(\frac{\Lambda(t)}{\Lambda(\tau)}\right)^{2}-\frac{\Lambda(\tau)}{\Lambda(t)}\right) \frac{d \alpha_{2}}{d \tau} d \tau \\
& +\mu_{5} \int_{t_{3}}^{t_{4}}\left(\left(\left(\frac{\Lambda(t)}{\Lambda(\tau)}\right)^{2}-1\right)\left(\frac{\Lambda(t)}{\Lambda(\tau)}\right)^{2}\right) \frac{d \alpha_{2}}{d \tau} d \tau
\end{aligned}
$$

Next we will derive equations for each stage of the triple shape memory process for the uni-axial cycle for the different TSCPs.

\subsection{TSCP-1}

\subsubsection{Loading-1}

During the first loading process, the temperature of the material is above both the transition temperatures and the material is entirely amorphous. Hence, using Eq.(23) the stress in the absence of crystallinity reduces to : 


$$
T_{11}=\left(\Lambda(t)^{2}-\frac{1}{\Lambda(t)}\right)
$$

For the case when the final stress is known, by prescribing the stress as a function of time, the progression of stretch is readily known from Eq.(24). Conversely, for the case when the final stretch is known, the algebraic equation for stress can be solved, by prescribing the stretch as a function of time.

\subsubsection{Crystallization-1}

Crystallization can be done either under constant strain or constant stress. The solution methodology is

slightly different, depending upon how the crystallization takes place. Both cases will be explained separately.

\subsubsection{Crystallization under constant strain}

When crystallization is done under constant strain, there is no change in the stretch i.e.:

$$
\Lambda(\tau)=\Lambda\left(t_{1}\right), \quad t_{1}<\tau<t<t_{2}
$$

where, $t_{1}$ is the time when crystallization of the first phase or phase- 1 at higher temperature is initiated, $\mathrm{t}$ is the current time, $t_{2}$ is the time at which crystallization of the first phase ends and $\tau$ is some intermediate time. Noting that the crystalline phase is formed in a stress free state and hence the contribution to the stress from the crystalline phase, while the stretch is kept constant, is zero and using Eq. (25) in Eq.(23) results in the following equation for the stress:

$$
T_{11}=\left(1-\alpha_{1_{H}}(t)\right)\left(\Lambda(t)^{2}-\frac{1}{\Lambda(t)}\right)
$$

The rate of crystallization is given by the appropriate crystallization kinetics, which are closely related to the temperature and thermodynamics of the problem. Barot et al. [44] have derived rate equations for shape memory polymers based on thermodynamic considerations. However, in this paper as we sought to clarify issues regarding the mechanical performance of triple shape memory polymers. We will not delve into the thermodynamics of the problem here that will be the topic of a future paper. Here, we assume that the crystallization rate equation gives the rate at which the crystallization takes place, with the tacit understanding that such an equation can be derived from a firm basis in thermodynamics (see Rao and Rajagopal [27], Barot et al. [44]). The specific equation that is chosen to mimic the rate of crystallization is given through a differential equation for the mass fraction of the crystalline phase and is given by:

$$
\alpha_{1_{H}}=0, \text { for } 0<t<t_{1}, \quad \frac{d \alpha_{1_{H}}}{d \tau_{1}}=G_{1}\left(t-t_{1}\right)\left(\alpha_{01_{H}}-\alpha_{1_{H}}\right), \text { for } t_{1}<t<t_{2}
$$

where, in the above equation $G_{1}$ is a constant, $\alpha_{01_{H}}$ is the maximum crystallinity of the phase- 1 that is possible in the material at the higher temperature and $t_{1}$ is the time at which crystallization is initiated at the higher temperature. The above equation is solved numerically using a standard numerical scheme for 
ordinary differential equations. Once crystallization ceases, i.e., when, $\alpha_{1_{H}}=\alpha_{01_{H}}$ at a later time denoted by $t_{2}$, the material is unloaded.

\subsubsection{Crystallization under constant stress}

The mechanical behavior is quite different when the polymer crystallizes under constant stress. The polymer stretches as the crystallization proceeds, as the newly crystallized material is formed in a stress free state, while the polymer is held under constant stress. The ability of the amorphous polymer to carry stress reduces when a part of it crystallizes, as there is less amorphous polymer available. However, the newly formed crystalline polymer is formed in a stress free state and is unable to carry the load either. The only way the polymer can sustain this stress is to stretch as the already present crystalline phase and the remaining amorphous phase take on additional stress. As the crystalline phase is a lot stiffer than the original amorphous phase the magnitude of the increase in stretch is much smaller than the original stretch (see [43]). The variation of stretch with time is obtained from Eq. (23). The crystallization rate equation is identical to the one chosen earlier. In absence of the second crystalline phase and the first phase crystallized at lower temperature Eq.(23) can be re-written as:

$$
T_{11}=\left(1-\alpha_{1_{H}}(t)\right)\left(\Lambda\left(t^{2}\right)-\frac{1}{\Lambda(t)}\right)+\mu_{1}(\Lambda(t))^{2} L_{11}-\mu_{1}\left(\frac{1}{\Lambda(t)}\right) L_{12}+\mu_{2}(\Lambda(t))^{4} L_{13}-\mu_{2}(\Lambda(t))^{2} L_{11}
$$

where, $L_{11}, L_{12}, L_{13}$ are integrals that are defined through

$$
L_{11}=\int_{t_{1}}^{t} \frac{1}{(\Lambda(\tau))^{2}} \frac{d \alpha_{1_{H}}}{d \tau} d \tau, L_{12}=\int_{t_{1}}^{t}(\Lambda(\tau)) \frac{d \alpha_{1_{H}}}{d \tau} d \tau, L_{13}=\int_{t_{1}}^{t} \frac{1}{(\Lambda(\tau))^{4}} \frac{d \alpha_{1_{H}}}{d \tau} d \tau,
$$

As all past values of stretch and crystallization rates are known the values of these integrals are known at time t. The current value of stretch $\Lambda(\mathrm{t})$ is solved for by numerically evaluating the values of the three integrals in Eq.(29) and substituting them in Eq.(28). The only unknown variable in Eq.(28) now is the current stretch $\Lambda(\mathrm{t})$, the resulting equation is a polynomial equation of order 5 , which is solved numerically utilizing the fact that out of the five possible solutions only one is physically possible. The conditions that the correct root had to be real and greater than unity (as the polymer is stretched), sufficed to identify the physically realizable solution for this problem.

\subsubsection{Unloading-1}

During unloading-1 the material is a mixture of two different phases, the first crystalline phase or phase-1 crystallized at higher temperature and the amorphous phase with each having different stress-free states. Hence, upon unloading even though the mixture will be stress free both phases will not unload to a stressfree state. The equation for stress during unloading reduces to:

$$
\mathrm{T}_{11}=\left(1-\alpha_{1_{H}}(t)\right)\left(\Lambda(t)^{2}-\frac{1}{\Lambda(t)}\right)+\mu_{1} \int_{t_{1}}^{t_{2}}\left(\left(\frac{\Lambda(t)}{\Lambda(\tau)}\right)^{2}-\frac{\Lambda(\tau)}{\Lambda(t)}\right) \frac{d \alpha_{1_{H}}}{d \tau} d \tau+\mu_{2} \int_{t_{1}}^{t_{2}}\left(\left(\left(\frac{\Lambda(t)}{\Lambda(\tau)}\right)^{2}-1\right)\left(\frac{\Lambda(t)}{\Lambda(\tau)}\right)^{2}\right) \frac{d \alpha_{1_{H}}}{d \tau} d \tau
$$


The limit of the integral in the above equation is now $t_{2}$, that is the time at which crystallization of the phase-1 ended. Again, re-writing Eq.(30) in an identical manner to Eqs. (28) and (29) we obtain:

$$
\begin{gathered}
\mathrm{T}_{11}=\left(1-\alpha_{1_{H}}(t)\right)\left(\Lambda(t)^{2}-\frac{1}{\Lambda(t)}\right)+\mu_{1}(\Lambda(t))^{2} L_{11}-\mu_{1}\left(\frac{1}{\Lambda(t)}\right)+\mu_{2}(\Lambda(t))^{4} L_{13}-\mu_{2}(\Lambda(t))^{2} L_{11} \\
L_{11}=\int_{t_{1}}^{t_{2}} \frac{1}{(\Lambda(\tau))^{2}} \frac{d \alpha_{1_{H}}}{d \tau} d \tau, L_{12}=\int_{t_{1}}^{t_{2}}(\Lambda(\tau)) \frac{d \alpha_{1_{H}}}{d \tau} d \tau, L_{13}=\int_{t_{1}}^{t_{2}} \frac{1}{(\Lambda(\tau))^{4}} \frac{d \alpha_{1_{H}}}{d \tau} d \tau,
\end{gathered}
$$

where, $L_{11}, L_{12}, L_{13}$ are integrals, identical to those given by Eq.(29) expect the upper limit is $t_{2}$. During the unloading process the values of these integrals remain unchanged as their integrands only depend on the stretches and crystallization rates during crystallization, i.e. between times $t_{1}$ and $t_{2}$. The variation of stretch during the unloading process is determined by stepping the stress down from its value at the end of crystallization to zero. The value of stretch can be obtained for each intermediate value of stress by solving the resulting polynomial Eq. (31) in a manner identical to that described earlier, keeping in mind that out of the multiple roots, the right root is the one that has physical significance.

\subsubsection{Loading-2}

During the second loading process, the material is below the first transition temperature $\left(T_{R_{1}}\right)$ and above the second transition temperature $\left(T_{R_{2}}\right)$. It consists of a mixture of amorphous phase and the first crystalline phase. Hence, using Eq.(23) the stress reduces to:

$$
\mathrm{T}_{11}=\left(1-\alpha_{1_{H}}(t)\right)\left(\Lambda(t)^{2}-\frac{1}{\Lambda(t)}\right)+\mu_{1}(\Lambda(t))^{2} L_{11}-\mu_{1}\left(\frac{1}{\Lambda(t)}\right) L_{12}+\mu_{2}(\Lambda(t))^{4} L_{13}-\mu_{2}(\Lambda(t))^{2} L_{11}
$$

where, $L_{11}, L_{12}, L_{13}$ are integrals that are identical to those given in Eq.(32), the values of these integrals remain unchanged during the loading 2 process as described earlier. Eq.(33) is solved for stretch or stress in a manner identical to unloading-1.

\subsubsection{Crystallization-2}

As the polymer is cooled below the second recovery temperature $T_{R_{2}}$, the second phase crystallizes. A portion of phase-1 that did not crystallize at the higher temperature also crystallizes during this process.

\subsubsection{Crystallization-2 under constant strain}

When crystallization is done under constant strain there is no change in stretch i.e.:

$$
\Lambda(\tau)=\Lambda\left(t_{3}\right), \quad t_{3}<\tau<t<t_{4}
$$

where, $t_{3}$ is the time when crystallization at lower temperature is initiated, $\mathrm{t}$ is the current time, $t_{4}$ is the time when crystallization at lower temperature ends and $\tau$ is some intermediate time. As explained 
earlier the newly formed crystalline phases under constant strain are formed in a stress free state and do not contribute to the stress. The equation for the stress reduces to:

$$
\begin{aligned}
& \mathrm{T}_{11}=\left(1-\alpha_{1_{H}}(t)-\alpha_{1_{L}}(t)-\alpha_{2}(t)\right)\left(\Lambda(t)^{2}-\frac{1}{\Lambda(t)}\right)+\mu_{1}(\Lambda(t))^{2} L_{11}-\mu_{1}\left(\frac{1}{\Lambda(t)}\right) L_{12} \\
& +\mu_{2}(\Lambda(t))^{4} L_{13}-\mu_{2}(\Lambda(t))^{2} L_{11}
\end{aligned}
$$

where, $L_{11}, L_{12}, L_{13}$ are integrals, that are identical to those given in Eq.(32), $\alpha_{1_{H}}$ is the maximum value of the first crystalline phase formed at the higher temperature. The values of the integrals remain unchanged during the second crystallization process, as their integrands only depend on the stresses and crystallization rates during crystallization of first phase at the higher temperature. Similar to crystallization-1 specific equations to mimic the rate of crystallization are given through differential equations for mass fraction of the crystalline phase and are given by:

$$
\begin{gathered}
\alpha_{2}=0, \text { for } 0<t<t_{3}, \frac{d \alpha_{2}}{d \tau}=G_{2}\left(t-t_{3}\right)\left(\alpha_{0_{2}}-\alpha_{2}\right), \text { for } t_{3}<t<t_{4} \\
\alpha_{1_{L}}=0, \text { for } 0<t<t_{3}, \frac{d \alpha_{1_{L}}}{d \tau}=G_{1}^{\prime}\left(t-t_{3}\right)\left(\alpha_{0_{1_{L}}}-\alpha_{1_{L}}\right), \text { for } t_{3}<t<t_{4}
\end{gathered}
$$

where, in the above equation $G_{2}$ and $G_{1}^{\prime}$ are constants, $\alpha_{01_{L}}$ is the maximum crystallinity of the first phase crystallizing at lower temperature, possible in the material, $\alpha_{0_{2}}$ is the maximum crystallinity of the second phase that crystallizes at the lower temperature possible in the material and $t_{3}$ is the time at which crystallization at the lower temperature is initiated. The above equations are solved in a manner identical to crystallization-1. Once crystallization ceases, i.e., when, $\alpha_{1_{L}}=\alpha_{01_{L}}$ and $\alpha_{2}=\alpha_{0_{2}}$ at a later time denoted by $t_{4}$, the material is unloaded.

\subsubsection{Crystallization-2 under constant stress}

During the crystallization process under constant stress the polymer will stretch as crystallization proceeds. The variation of the stretch with time is obtained from Eq.(23). The equations for the crystallization rates of the second phase and the first phase crystallizing at the lower temperature are identical to the one chosen earlier. Eq.(23) can be re-written as: 


$$
\begin{aligned}
& \mathrm{T}_{11}=\left(1-\alpha_{1_{H}}(t)-\alpha_{1_{L}}(t)-\alpha_{2}(t)\right)\left(\Lambda(t)^{2}-\frac{1}{\Lambda(t)}\right)+\mu_{1}(\Lambda(t))^{2} L_{11}-\mu_{1}\left(\frac{1}{\Lambda(t)}\right) L_{12} \\
& +\mu_{2}(\Lambda(t))^{4} L_{13}-\mu_{2}(\Lambda(t))^{2} L_{11}+\mu_{1}^{\prime}(\Lambda(t))^{2} L_{31}-\mu_{1}^{\prime}\left(\frac{1}{\Lambda(t)}\right) L_{32} \\
& +\mu_{2}^{\prime}(\Lambda(t))^{4} L_{33}-\mu_{2}^{\prime}(\Lambda(t))^{2} L_{31}+\mu_{4}(\Lambda(t))^{2} L_{21}-\mu_{4}\left(\frac{1}{\Lambda(t)}\right) L_{22} \\
& +\mu_{5}(\Lambda(t))^{4} L_{23}-\mu_{5}(\Lambda(t))^{2} L_{21}
\end{aligned}
$$

where, $L_{11}, L_{12}, L_{13}$ are integrals, that are identical to those given in Eq.(32) and $L_{21}, L_{22}, L_{23}, L_{31}, L_{32}, L_{33}$ are integrals that are defined through,

$$
\begin{aligned}
& L_{31}=\int_{t_{3}}^{t} \frac{1}{(\Lambda(\tau))^{2}} \frac{d \alpha_{1_{L}}}{d \tau} d \tau, L_{32}=\int_{t_{3}}^{t}(\Lambda(\tau)) \frac{d \alpha_{1_{L}}}{d \tau} d \tau, L_{33}=\int_{t_{3}}^{t} \frac{1}{(\Lambda(\tau))^{4}} \frac{d \alpha_{1_{L}}}{d \tau} d \tau, \\
& L_{21}=\int_{t_{3}}^{t} \frac{1}{(\Lambda(\tau))^{2}} \frac{d \alpha_{2}}{d \tau} d \tau, L_{22}=\int_{t_{3}}^{t}(\Lambda(\tau)) \frac{d \alpha_{2}}{d \tau} d \tau, L_{23}=\int_{t_{3}}^{t} \frac{1}{(\Lambda(\tau))^{4}} \frac{d \alpha_{2}}{d \tau} d \tau,
\end{aligned}
$$

The values of the integrals $L_{11}, L_{12}, L_{13}$ remain unchanged during crystallization at the lower temperature. As the values of these integrands only depend on the stretches and crystallization rates during the crystallization of the first phase at the higher transition temperature, i.e. between times $t_{1}$ and $t_{2}$. The values of the integrals $L_{21}, L_{22}, L_{23}, L_{31}, L_{32}, L_{33}$ are known at time $\mathrm{t}$ as all past values of stretch and crystallization rates are known. To solve for the current value of stretch similar solution procedure as described earlier for the constant stress case during crystallization-1 is adopted.

\subsubsection{Unloading-2}

During unloading-2, the material is a mixture of different phases, the amorphous phase, the first crystalline phase crystallized during the first cooling process below the higher transition temperature $T_{R_{1}}$, the second crystalline phase and the first crystalline phase crystallized during the second cooling process below the lower transition temperature $T_{R_{2}}$. Each of the different phases have different stress-free states, hence they will not unload to a stress free state even though the mixture is stress free. The equation for the stress during unloading-2 reduces to: 


$$
\begin{aligned}
& \mathrm{T}_{11}=\left(1-\alpha_{1_{H}}(t)-\alpha_{1_{L}}(t)-\alpha_{2}(t)\right)\left(\Lambda(t)^{2}-\frac{1}{\Lambda(t)}\right)+\mu_{1}(\Lambda(t))^{2} L_{11}-\mu_{1}\left(\frac{1}{\Lambda(t)}\right) L_{12} \\
& +\mu_{2}(\Lambda(t))^{4} L_{13}-\mu_{2}(\Lambda(t))^{2} L_{11}+\mu_{1}^{\prime}(\Lambda(t))^{2} L_{31}-\mu_{1}^{\prime}\left(\frac{1}{\Lambda(t)}\right) L_{32} \\
& +\mu_{2}^{\prime}(\Lambda(t))^{4} L_{33}-\mu_{2}^{\prime}(\Lambda(t))^{2} L_{31}+\mu_{4}(\Lambda(t))^{2} L_{21}-\mu_{4}\left(\frac{1}{\Lambda(t)}\right) L_{22} \\
& +\mu_{5}(\Lambda(t))^{4} L_{23}-\mu_{5}(\Lambda(t))^{2} L_{21}
\end{aligned}
$$

where, $L_{11}, L_{12}, L_{13}$ are integrals, that are identical to those given in Eq.(32) and $L_{21}, L_{22}, L_{23}, L_{31}, L_{32}, L_{33}$ are integrals that are defined through,

$$
\begin{aligned}
& L_{31}=\int_{t_{3}}^{t_{4}} \frac{1}{(\Lambda(\tau))^{2}} \frac{d \alpha_{1_{L}}}{d \tau} d \tau, L_{32}=\int_{t_{3}}^{t_{4}}(\Lambda(\tau)) \frac{d \alpha_{1_{L}}}{d \tau} d \tau, L_{33}=\int_{t_{3}}^{t_{4}} \frac{1}{(\Lambda(\tau))^{4}} \frac{d \alpha_{1_{L}}}{d \tau} d \tau, \\
& L_{21}=\int_{t_{3}}^{t_{4}} \frac{1}{(\Lambda(\tau))^{2}} \frac{d \alpha_{2}}{d \tau} d \tau, L_{22}=\int_{t_{3}}^{t_{4}}(\Lambda(\tau)) \frac{d \alpha_{2}}{d \tau} d \tau, L_{23}=\int_{t_{3}}^{t_{4}} \frac{1}{(\Lambda(\tau))^{4}} \frac{d \alpha_{2}}{d \tau} d \tau,
\end{aligned}
$$

Integrals $L_{21}, L_{22}, L_{23}, L_{31}, L_{32}, L_{33}$ are identical to those given by Eq.(39) except the upper limit is $t_{4}$. The values of these integrals remain unchanged during the unloading-2 process as their integrands only depend on the stretches and crystallization rates during the crystallization below the lower transition temperature $T_{R_{2}}$, i.e. between times $t_{3}$ and $t_{4}$. The value of stretch during unloading-2 is determined in a manner similar to unloading-1.

\subsubsection{Melting-1}

After unloading-2 the polymer is in its second temporary shape. The two distinct temporary shapes of the polymer are retained because the different phases present have different stress free states. The second temporary shape is fixed by the second crystalline phase and the first crystalline phase that crystallizes at the lower temperature. On heating the polymer above the melting temperature of the second crystalline phase, the second crystalline phase melts. As it melts the shape of the polymer slowly returns towards the first temporary shape as the second crystalline phase is no longer present to keep it in the second temporary shape. It should be noted here that the portion of first phase that crystallized at the lower temperature does not melt during this melting process. As a result the first temporary shape is not recovered completely during TSCP-1. It is necessary to track which fraction of the second crystalline phase is melting at any given time in order to track the evolution of the shape as it evolves from the second temporary shape to the first temporary shape. For the case when crystallization took place under constant stress, this is particularly true, as different crystals have different stress free states as they are formed at different stretches. So the polymer as a whole will retract towards its first temporary shape more if the crystallite of the second phase that is more stressed melts than if a less stressed crystallite melted. For the constant stretch case the order in which the crystals melt does not impact the intermediate shapes occupied by the polymer as all the crystals were formed in the same configuration. However, for crystallization under constant stress, the order in which the crystallites melt is important. The assumption 
we make with regards to the melting process is that the crystallites formed last melt first, this assumption is supported from experiments as crystallites formed furthest from the equilibrium melting temperature ( i.e. crystallites that were formed last) are thinner and melt at lower temperatures ( i.e. melt first). During the melting-1 process, the second crystalline phase begins to melt at some time denoted in this paper by $t_{5}$. The stress in the polymer is zero and the equation for determining the stretch simplifies to:

$$
\begin{aligned}
& \mathrm{T}_{11}=0=\left(1-\alpha_{1_{H}}(t)-\alpha_{1_{L}}(t)-\alpha_{2}(t)\right)\left(\Lambda(t)^{2}-\frac{1}{\Lambda(t)}\right)+\mu_{1} \int_{t_{1}}^{t_{2}}\left(\left(\frac{\Lambda(t)}{\Lambda(\tau)}\right)^{2}-\frac{\Lambda(\tau)}{\Lambda(t)}\right) \frac{d \alpha_{1_{H}}}{d \tau} d \tau \\
& \mu_{2} \int_{t_{1}}^{t_{2}}\left(\left(\left(\frac{\Lambda(t)}{\Lambda(\tau)}\right)^{2}-1\right)\left(\frac{\Lambda(t)}{\Lambda(\tau)}\right)^{2}\right) \frac{d \alpha_{1_{H}}}{d \tau} d \tau+\mu_{1}^{\prime} \int_{t_{3}}^{t_{4}}\left(\left(\frac{\Lambda(t)}{\Lambda(\tau)}\right)^{2}-\frac{\Lambda(\tau)}{\Lambda(t)}\right) \frac{d \alpha_{1_{L}}}{d \tau} d \tau \\
& +\mu_{2}^{\prime} \int_{t_{3}}^{t_{4}}\left(\left(\left(\frac{\Lambda(t)}{\Lambda(\tau)}\right)^{2}-1\right)\left(\frac{\Lambda(t)}{\Lambda(\tau)}\right)^{2}\right) \frac{d \alpha_{1_{L}}}{d \tau} d \tau+\mu_{4} \int_{t_{3}}^{t^{* *}}\left(\left(\frac{\Lambda(t)}{\Lambda(\tau)}\right)^{2}-\frac{\Lambda(\tau)}{\Lambda(t)}\right) \frac{d \alpha_{2}}{d \tau} d \tau \\
& +\mu_{5} \int_{t_{3}}^{t^{*}}\left(\left(\left(\frac{\Lambda(t)}{\Lambda(\tau)}\right)^{2}-1\right)\left(\frac{\Lambda(t)}{\Lambda(\tau)}\right)^{2}\right) \frac{d \alpha_{2}}{d \tau} d \tau
\end{aligned}
$$

where, $t^{* *} \in\left[\mathrm{t}_{3}, \mathrm{t}_{4}\right]$ and $\alpha_{2}\left(\mathrm{t}^{* *}\right)=\alpha_{2}(\mathrm{t})$

Note, in the above equation we are tagging the time when the crystals of the second phase were formed to the actual times when they were formed, i.e. $\tau \in\left[\mathrm{t}_{3}, \mathrm{t}_{4}\right]$. However, after melting of the second phase begins, a part of the original crystals that were present have melted and converted back to an amorphous phase. The current value of crystallinity of the second phase, i.e., the amount that has melted is known from the rate equation for melting of the second phase, which will be described in detail in the later section.

Since crystallinity of the second phase is tracked as a function of time during crystallization, we can determine the time during the crystallization process, denoted by $t^{* *} \in\left[\mathrm{t}_{3}, \mathrm{t}_{4}\right]$, when the amount of second phase or phase- 2 crystalline material present equals the current levels of crystallinity during melting, i.e., $\alpha_{2}\left(\mathrm{t}^{* *}\right)=\alpha_{2}(\tau)$, through interpolation between known values of crystallinity and the times at which those values occur. This is possible because of the assumption that the crystallites formed last melt first. Re-writing Eq.(42) as: 


$$
\begin{aligned}
& \mathrm{T}_{11}=\left(1-\alpha_{1_{H}}(t)-\alpha_{1_{L}}(t)-\alpha_{2}(t)\right)\left(\Lambda(t)^{2}-\frac{1}{\Lambda(t)}\right)+\mu_{1}(\Lambda(t))^{2} L_{11}-\mu_{1}\left(\frac{1}{\Lambda(t)}\right) L_{12} \\
& +\mu_{2}(\Lambda(t))^{4} L_{13}-\mu_{2}(\Lambda(t))^{2} L_{11}+\mu_{1}^{\prime}(\Lambda(t))^{2} L_{31}-\mu_{1}^{\prime}\left(\frac{1}{\Lambda(t)}\right) L_{32} \\
& +\mu_{2}^{\prime}(\Lambda(t))^{4} L_{33}-\mu_{2}^{\prime}(\Lambda(t))^{2} L_{31}+\mu_{4}(\Lambda(t))^{2} L_{21}-\mu_{4}\left(\frac{1}{\Lambda(t)}\right) L_{22} \\
& +\mu_{5}(\Lambda(t))^{4} L_{23}-\mu_{5}(\Lambda(t))^{2} L_{21}
\end{aligned}
$$

where, $L_{21}, L_{22}, L_{23}, L_{31}, L_{32}, L_{33}$ are integrals with integrands identical to Eq.(32) and Eq.(41) and integrals $L_{11}, L_{12}, L_{13}$ have integrands identical to Eq.(41) with different limits and are given by:

$$
L_{21}=\int_{t_{3}}^{t_{3}^{* *}} \frac{1}{(\Lambda(\tau))^{2}} \frac{d \alpha_{2}}{d \tau} d \tau, L_{22}=\int_{t_{3}}^{t_{*}^{* *}}(\Lambda(\tau)) \frac{d \alpha_{2}}{d \tau} d \tau, L_{23}=\int_{t_{3}}^{t_{3}^{* *}} \frac{1}{(\Lambda(\tau))^{4}} \frac{d \alpha_{2}}{d \tau} d \tau
$$

Incremental values of crystallinity of phase- 2 are obtained from the prescribed melting equation for crystallinity, which is given by:

$$
\frac{d \alpha_{2}}{d \tau}=G_{2}\left(t-t_{5}\right)\left(\alpha_{0_{2}}-\alpha_{2}\right), \quad \text { for } t_{5}<t<t_{6}
$$

where, in the above equation $G_{2}$ is a constant, $t_{5}$ is the time at which melting of the second phase is initiated by heating over second transition temperature $T_{R_{2}}$ and $t_{6}$ is the time at which melting of the second phase ceases, i.e. when $\alpha_{2}=0$. Here we are prescribing the melting rate equation for the second phase, as we are mainly focusing on the mechanical implications. Solving Eq.(45) we can determine the incremental decrease in the crystallinity of the second phase and its net crystallinity after each time step. Then using the interpolation procedure described above we determine $t^{* *}$ and hence evaluate the integrals in Eq.(44). The values of the integrals are substituted into Eq.(43) and the resulting polynomial equation is solved for the appropriate value of stretch.

\subsubsection{Melting-2}

After melting-1 the polymer consists of the amorphous phase, first phase crystallized at higher temperature and the portion of first phase that crystallizes at a lower temperature. The temporary shapes are retained because each phase has a different stress free state. On heating above the first transition temperature $T_{R_{1}}$, the first crystalline phase that crystallized both at the higher temperature $\left(\alpha_{1_{H}}\right)$ and lower temperature $\left(\alpha_{1_{L}}\right)$ melts. As these crystalline phases melt the polymer slowly retracts to its original shape as the crystalline phase is no longer present to keep it in its temporary shape. Again as discussed earlier to track the evolution of shape we use the assumption that the crystallites formed last melt first. Therefore the portion of first phase that crystallized at the lower temperature melts first followed by the first phase that had crystallized at higher temperature. 
The stress in the polymer is zero and the equation for determining the stretch simplifies to:

$$
\begin{aligned}
& \mathrm{T}_{11}=0=\left(1-\alpha_{1_{H}}(t)-\alpha_{1_{L}}(t)\right)\left(\Lambda(t)^{2}-\frac{1}{\Lambda(t)}\right)+\mu_{1} \int_{t_{1}}^{t^{*}}\left(\left(\frac{\Lambda(t)}{\Lambda(\tau)}\right)^{2}-\frac{\Lambda(\tau)}{\Lambda(t)}\right) \frac{d \alpha_{1_{H}}}{d \tau} d \tau \\
& \mu_{2} \int_{t_{1}}^{t^{*}}\left(\left(\left(\frac{\Lambda(t)}{\Lambda(\tau)}\right)^{2}-1\right)\left(\frac{\Lambda(t)}{\Lambda(\tau)}\right)^{2}\right) \frac{d \alpha_{1_{H}}}{d \tau} d \tau+\mu_{1}^{\prime} \int_{t_{3}}^{t^{* *}}\left(\left(\frac{\Lambda(t)}{\Lambda(\tau)}\right)^{2}-\frac{\Lambda(\tau)}{\Lambda(t)}\right) \frac{d \alpha_{1_{L}}}{d \tau} d \tau \\
& +\mu_{2}^{\prime} \int_{t_{3}}^{t^{*}}\left(\left(\left(\frac{\Lambda(t)}{\Lambda(\tau)}\right)^{2}-1\right)\left(\frac{\Lambda(t)}{\Lambda(\tau)}\right)^{2}\right) \frac{d \alpha_{1_{L}}}{d \tau} d \tau
\end{aligned}
$$

where, $t^{\prime *} \in\left[t_{3}, t_{4}\right], t^{*} \in\left[t_{1}, t_{2}\right]$ and $\alpha_{1_{L}}\left(t^{\prime *}\right)=\alpha_{1_{L}}(t), \alpha_{1_{H}}\left(t^{*}\right)=\alpha_{1_{H}}(t)$

Again, we are tagging time when the crystals were formed to the actual times when they were formed, i.e. $\tau \in\left[\mathrm{t}_{1}, \mathrm{t}_{2}\right], \tau \in\left[\mathrm{t}_{3}, \mathrm{t}_{4}\right]$. From rate equations for melting of the first phase formed at both higher and lower temperatures, current value of the crystallinity is known, i.e., the amount that has melted is also known. Similar to melting-1 we track crystallinity as a function of time during crystallization, by interpolation between known values of crystallinity of the first phase crystallized both at the higher and lower temperatures and the times at which those values occur, we can determine the time during the crystallization processes, denoted by $t^{\prime *} \in\left[t_{3}, t_{4}\right], t^{*} \in\left[t_{1}, t_{2}\right]$, when the amount of the crystalline material present equals the current level of crystallinity during the melting, i.e., $\alpha_{1_{L}}\left(t^{\prime *}\right)=\alpha_{1_{L}}(t), \alpha_{1_{H}}\left(t^{*}\right)=\alpha_{1_{H}}(t)$. Again, this is possible due to the assumption that the crystallites formed last melt first. Re-writing Eq.(46) as:

$$
\begin{aligned}
& \mathrm{T}_{11}=\left(1-\alpha_{1_{H}}(t)-\alpha_{1_{L}}(t)\right)\left(\Lambda(t)^{2}-\frac{1}{\Lambda(t)}\right)+\mu_{1}(\Lambda(t))^{2} L_{11}-\mu_{1}\left(\frac{1}{\Lambda(t)}\right) L_{12} \\
& +\mu_{2}(\Lambda(t))^{4} L_{13}-\mu_{2}(\Lambda(t))^{2} L_{11}+\mu_{1}^{\prime}(\Lambda(t))^{2} L_{31}-\mu_{1}^{\prime}\left(\frac{1}{\Lambda(t)}\right) L_{32} \\
& +\mu_{2}^{\prime}(\Lambda(t))^{4} L_{33}-\mu_{2}^{\prime}(\Lambda(t))^{2} L_{31}
\end{aligned}
$$

where, integrals $L_{11}, L_{12}, L_{13}, L_{31}, L_{32}, L_{33}$ are given by:

$$
\begin{aligned}
& L_{11}=\int_{t_{1}}^{t^{*}} \frac{1}{(\Lambda(\tau))^{2}} \frac{d \alpha_{1_{H}}}{d \tau} d \tau, L_{12}=\int_{t_{1}}^{t^{*}}(\Lambda(\tau)) \frac{d \alpha_{1_{H}}}{d \tau} d \tau, L_{13}=\int_{t_{1}}^{t^{*}} \frac{1}{(\Lambda(\tau))^{4}} \frac{d \alpha_{1_{H}}}{d \tau} d \tau, \\
& L_{31}=\int_{t_{3}}^{t^{*}} \frac{1}{(\Lambda(\tau))^{2}} \frac{d \alpha_{1_{L}}}{d \tau} d \tau, L_{32}=\int_{t_{3}}^{t^{t^{*}}}(\Lambda(\tau)) \frac{d \alpha_{1_{L}}}{d \tau} d \tau, L_{33}=\int_{t_{3}}^{t^{*^{*}}} \frac{1}{(\Lambda(\tau))^{4}} \frac{d \alpha_{1_{L}}}{d \tau} d \tau,
\end{aligned}
$$


Similar to melting-1 incremental values of crystallinity of the first crystalline phase crystallized at both the higher and lower temperatures are obtained from the prescribed melting equations for crystallinity, which are given by:

$$
\begin{array}{cl}
\frac{d \alpha_{1_{L}}}{d \tau}=G_{1}\left(t-t_{7}\right)\left(\alpha_{0_{1_{L}}}-\alpha_{1_{L}}\right), & \text { for } t_{7}<t<t_{8} \\
\frac{d \alpha_{1_{H}}}{d \tau}=G_{1}\left(t-t_{9}\right)\left(\alpha_{0_{1_{H}}}-\alpha_{1_{H}}\right), & \text { for } t_{9}<t<t_{10}
\end{array}
$$

where, in the above equation $G_{1}^{\prime}$ and $G_{1}$ are constants, $t_{7}$ is the time at which melting of the portion of first phase formed at the lower temperature is initiated and $t_{8}$ is the time at which melting of the first phase formed at the lower temperature ceases, i.e. when $\alpha_{1_{L}}=0$ and $t_{9}$ is the time at which melting of the first phase formed at the higher temperature is initiated and $t_{10}$ is the time at which melting of the first phase formed at the higher temperature ceases, i.e. when $\alpha_{1_{H}}=0$. Solving Eq.(49) and Eq.(50) we can determine the incremental decrease in the crystallinity of the first phase crystallized at the low temperature and the high temperature respectively and the net crystallinity after each time step. Then using the interpolation procedure described above we determine $t^{\prime *}$ and $t^{*}$, and hence evaluate the integrals in Eq.(48). The values of the integrals are substituted into Eq.(47) and the resulting polynomial equation is solved for the appropriate value of stretch.

\subsection{TSCP-2}

The second triple shape creation process TSCP-2 consists of nine distinct phases Loading-1, crystallization-1, unloading-1, melting-1, loading-2, crystallization-2, unloading-3, melting-2, melting-3. To capture the complete behavior the entire cycle can be modeled, and equations for each stage can be derived by proceeding in a manner similar to TSCP-1. Equations for each stage of TCSP-2 are derived next. During loading-1 stress takes exactly the same form as Eq.(24), solution procedure similar to TSCP-1 is adopted.

\subsubsection{Crystallization-1}

Both cases for crystallization under constant strain and constant stress are explained separately.

\subsubsection{Crystallization-1 under constant strain}

There is no change in stretch during crystallization under constant strain i.e.:

$$
\begin{gathered}
\Lambda(\tau)=\Lambda\left(t_{1}\right), \quad t_{1}<\tau<t<t_{2} \\
\Lambda(\tau)=\Lambda\left(t_{3}\right), \quad t_{2}<t_{3}<\tau<t<t_{4}
\end{gathered}
$$

where, $t_{1}$ is the time when crystallization of the first phase is initiated at the higher temperature, $\tau$ is some intermediate time, $t_{2}$ is the time when crystallization of the first phase ends. At the lower 
temperature some part of the first phase crystallizes along with the second phase as explained earlier, $t_{3}$ is the time when crystallization is initiated at the lower temperature $\tau$ is some intermediate time, $t_{4}$ is the time when crystallization at the lower temperature ends, $t$ is the current time. Proceeding in a manner similar as the previous case, results in the following equation for stress:

$$
\mathrm{T}_{11}=\left(1-\alpha_{1_{H}}(t)-\alpha_{1_{L}}(t)-\alpha_{2}(t)\right)\left(\Lambda(t)^{2}-\frac{1}{\Lambda(t)}\right)
$$

The crystallization rate equations for all the crystalline phases formed are identical to the ones used in TSCP-1.

\subsubsection{Crystallization-1 under constant stress}

As described earlier, the polymer will stretch as the crystallization proceeds under constant stress. Equation for stress during crystallization of the first phase at higher temperature is identical to Eq. (28). Equation for stress during crystallization of the second phase and the first phase at lower temperature is identical to Eq.(38). The values of the integrals $L_{11}, L_{12}, L_{13}$ remain unchanged during crystallization at the lower temperature, as described earlier. To solve for the current value of stretch, solution procedure similar to TSCP-1 is adopted.

\subsubsection{Unloading-1}

Unlike TSCP-1 during unloading-1 the material is a mixture of amorphous phase, crystalline phase-1 formed at both higher and lower temperatures and crystalline phase-2. The equation for stress during unloading-1 can be shown to reduce to Eq.(40). Solution procedure adopted to solve for the integrals and for the current value of stretch is identical to TSCP-1.

\subsubsection{Melting-1}

As the polymer is heated above the second recovery temperature during melting-1, the second phase melts. The melting rate equation for the incremental values of the second phase is identical to Eq.(45), where $t_{5}$ is the time at which melting of the second phase is initiated by heating over second transition temperature $T_{R_{2}}$ and $t_{6}$ is the time at which melting of the second phase ceases, i.e. when $\alpha_{2}=0$. The equation for stress is identical to Eq.(43), this equation can be solved for the current value of stretch in a manner that is identical to TSCP-1.

\subsubsection{Loading- 2}

During the second loading process, the material consists of a mixture of amorphous phase and the first crystalline phase that has crystallized at a higher temperature and at a lower temperature. Hence, using Eq. (23) the stress reduces to: 


$$
\begin{aligned}
& \mathrm{T}_{11}=\left(1-\alpha_{1_{H}}(t)-\alpha_{1_{L}}(t)\right)\left(\Lambda(t)^{2}-\frac{1}{\Lambda(t)}\right)+\mu_{1}(\Lambda(t))^{2} L_{11}-\mu_{1}\left(\frac{1}{\Lambda(t)}\right) L_{12} \\
& +\mu_{2}(\Lambda(t))^{4} L_{13}-\mu_{2}(\Lambda(t))^{2} L_{11}+\mu_{1}^{\prime}(\Lambda(t))^{2} L_{31}-\mu_{1}^{\prime}\left(\frac{1}{\Lambda(t)}\right) L_{32} \\
& +\mu_{2}^{\prime}(\Lambda(t))^{4} L_{33}-\mu_{2}^{\prime}(\Lambda(t))^{2} L_{31}
\end{aligned}
$$

where, $L_{11}, L_{12}, L_{13}, L_{31}, L_{32}, L_{33}$ are integrals that are defined through,

$$
\begin{aligned}
& L_{11}=\int_{t_{1}}^{t_{2}} \frac{1}{(\Lambda(\tau))^{2}} \frac{d \alpha_{1_{H}}}{d \tau} d \tau, L_{12}=\int_{t_{1}}^{t_{2}}(\Lambda(\tau)) \frac{d \alpha_{1_{H}}}{d \tau} d \tau, L_{13}=\int_{t_{1}}^{t_{2}} \frac{1}{(\Lambda(\tau))^{4}} \frac{d \alpha_{1_{H}}}{d \tau} d \tau, \\
& L_{31}=\int_{t_{3}}^{t_{4}} \frac{1}{(\Lambda(\tau))^{2}} \frac{d \alpha_{1_{L}}}{d \tau} d \tau, L_{32}=\int_{t_{3}}^{t_{4}}(\Lambda(\tau)) \frac{d \alpha_{1_{L}}}{d \tau} d \tau, L_{33}=\int_{t_{3}}^{t_{4}} \frac{1}{(\Lambda(\tau))^{4}} \frac{d \alpha_{1_{L}}}{d \tau} d \tau,
\end{aligned}
$$

The values of these integrals remain unchanged during the loading-2 process as discussed earlier. Eq.(54) is solved for stretch or stress.

\subsubsection{Crystallization-2}

During this process the polymer is cooled again below the second recovery temperature and the second phase crystallizes again.

\subsubsection{Crystallization-2 under constant strain}

There is no change in stretch when crystallization is done under constant strain i.e.:

$$
\begin{gathered}
\Lambda(\tau)=\Lambda\left(t_{7}\right), \quad t_{7}<\tau<t<t_{8} \\
t_{1}<t_{2}<t_{3}<t_{4}<t_{5}<t_{6}<t_{7}<t_{8}
\end{gathered}
$$

where, $t_{7}$ is the time when crystallization process is initiated at the lower temperature during the second cooling process, $\mathrm{t}$ is the current time, $\tau$ is some intermediate time and $t_{8}$ is the time when crystallization of phase- 2 ends. Noting that crystalline phase formed in a stress free state does not contribute to the stress, the equation for stress reduces to an equation similar to Eq.(54), only difference being crystalline phase- 2 is deducted from the amorphous phase. Crystallization rate used for the second phase can be given by Eq.(36) replacing $t_{3}, t_{4}$ with $t_{7}, t_{8}$ respectively.

\subsubsection{Crystallization- 2 under constant stress}

As discussed earlier, the polymer stretches during crystallization under constant stress. The crystallization rate is identical to the one used earlier. The stress in the polymer reduces to: 


$$
\begin{aligned}
& \mathrm{T}_{11}=\left(1-\alpha_{1_{H}}(t)-\alpha_{1_{L}}(t)-\alpha_{2}(t)\right)\left(\Lambda(t)^{2}-\frac{1}{\Lambda(t)}\right)+\mu_{1}(\Lambda(t))^{2} L_{11}-\mu_{1}\left(\frac{1}{\Lambda(t)}\right) L_{12} \\
& +\mu_{2}(\Lambda(t))^{4} L_{13}-\mu_{2}(\Lambda(t))^{2} L_{11}+\mu_{1}^{\prime}(\Lambda(t))^{2} L_{31}-\mu_{1}^{\prime}\left(\frac{1}{\Lambda(t)}\right) L_{32} \\
& +\mu_{2}^{\prime}(\Lambda(t))^{4} L_{33}-\mu_{2}^{\prime}(\Lambda(t))^{2} L_{31}+\mu_{4}(\Lambda(t))^{2} L_{21}-\mu_{4}\left(\frac{1}{\Lambda(t)}\right) L_{22} \\
& +\mu_{5}(\Lambda(t))^{4} L_{23}-\mu_{5}(\Lambda(t))^{2} L_{21}
\end{aligned}
$$

where, $L_{11}, L_{12}, L_{13}, L_{31}, L_{32}, L_{33}$ are integrals that can be given by Eq.(55), and $L_{21}, L_{22}, L_{23}$ are integrals that are defined through,

$$
L_{21}=\int_{t_{7}}^{t} \frac{1}{(\Lambda(\tau))^{2}} \frac{d \alpha_{2}}{d \tau} d \tau, L_{22}=\int_{t_{7}}^{t}(\Lambda(\tau)) \frac{d \alpha_{2}}{d \tau} d \tau, L_{23}=\int_{t_{7}}^{t} \frac{1}{(\Lambda(\tau))^{4}} \frac{d \alpha_{2}}{d \tau} d \tau
$$

Eq.(57) is solved for the current value of stretch.

\subsubsection{Unloading-3}

During unloading-3 the material is a mixture of different phases, after unloading the material is stress-free but the different phases do not unload to a stress free state as discussed earlier. The equation for stress during unloading is identical to Eq.(43). Where, $L_{11}, L_{12}, L_{13}, L_{31}, L_{32}, L_{33}$ are integrals that can be given by Eq.(55), and $L_{21}, L_{22}, L_{23}$ are integrals that are identical to Eq.(58) except the upper limit is given by $t_{8}$.

\subsubsection{Melting-2}

After unloading-3 the polymer is in its second temporary shape. Return to the first temporary shape is accomplished by melting of the second crystalline phase, by heating above its melting temperature. Using the assumption used in TSCP-1, that the crystallites formed last melt first, the equation to determine the stretch simplifies to:

$$
\begin{aligned}
& \mathrm{T}_{11}=\left(1-\alpha_{1_{H}}(t)-\alpha_{1_{L}}(t)-\alpha_{2}(t)\right)\left(\Lambda(t)^{2}-\frac{1}{\Lambda(t)}\right)+\mu_{1}(\Lambda(t))^{2} L_{11}-\mu_{1}\left(\frac{1}{\Lambda(t)}\right) L_{12} \\
& +\mu_{2}(\Lambda(t))^{4} L_{13}-\mu_{2}(\Lambda(t))^{2} L_{11}+\mu_{1}^{\prime}(\Lambda(t))^{2} L_{31}-\mu_{1}^{\prime}\left(\frac{1}{\Lambda(t)}\right) L_{32} \\
& +\mu_{2}^{\prime}(\Lambda(t))^{4} L_{33}-\mu_{2}^{\prime}(\Lambda(t))^{2} L_{31}+\mu_{4}(\Lambda(t))^{2} L_{21}-\mu_{4}\left(\frac{1}{\Lambda(t)}\right) L_{22} \\
& +\mu_{5}(\Lambda(t))^{4} L_{23}-\mu_{5}(\Lambda(t))^{2} L_{21}
\end{aligned}
$$


where, $L_{11}, L_{12}, L_{13}, L_{31}, L_{32}, L_{33}$ are integrals that can be given by Eq.(55), and $L_{21}, L_{22}, L_{23}$ are integrals that are defined through,

$$
L_{21}=\int_{t_{5}}^{t^{* * * *}} \frac{1}{(\Lambda(\tau))^{2}} \frac{d \alpha_{2}}{d \tau} d \tau, L_{22}=\int_{t_{5}}^{t^{* * * *}}(\Lambda(\tau)) \frac{d \alpha_{2}}{d \tau} d \tau, L_{23}=\int_{t_{5}}^{t^{* * * *}} \frac{1}{(\Lambda(\tau))^{4}} \frac{d \alpha_{2}}{d \tau} d \tau
$$

where, $t^{* * *} \in\left[t_{7}, t_{8}\right]$ and $\alpha_{2}\left(t^{* * * *}\right)=\alpha_{2}(t)$

Proceeding in a manner similar to the melting processes for TSCP-1 we are tagging the time when the crystals were formed to the actual times when they were formed, i.e. $\tau \in\left[\mathrm{t}_{7}, \mathrm{t}_{8}\right]$. Melting rate used is identical to the one used for TSCP-1, Eq.(59) is solved for the current value of stretch.

\subsubsection{Melting-3}

After Melting-2 the polymer is in its first temporary shape, return to the original shape is accomplished by melting the first crystalline phase that was crystallized both at a higher temperature and at a lower temperature. This is done by heating the polymer above the first recovery temperature. Using solution procedure similar to Melting-2 for TSCP-1 the equation for stress can be shown to be given by Eq.(47). Again we are tagging time when the crystals were formed to the actual times when they were formed, i.e. $\tau \in\left[\mathrm{t}_{1}, \mathrm{t}_{2}\right], \tau \in\left[\mathrm{t}_{3}, \mathrm{t}_{4}\right]$. This completes the solution procedure for TSCP-2 uni-axial deformation cycle.

\subsection{TSCP-3}

In TSCP-3 the TSMP is subjected to a constant stress throughout the triple shape memory cycle. As the polymer is stimulated by cyclic temperature changes it is able to switch between three shapes, the original shape, a first temporary shape and a second temporary shape. This TSCP consists of five phases Loading, Crystallization-1, Crystallization-2, Melting-1, and Melting-2. Proceeding in a manner similar to the previous cases equations for each stage of this TSCP can be derived. During the loading process stress can be shown to be given by Eq.(24). During the Crystallization-1 process crystallization takes place under constant stress. As the polymer is cooled below the first recovery temperature the first phase crystallizes. The stress in the polymer can be shown to reduce to Eq.(28), this equation is solved for the change in stretch as crystallization proceeds. The crystallization rate used is identical to Eq.(27). During crystallization- 2 as the polymer is cooled below the lower recovery temperature, along with the second phase some portion of the first phase that did not crystallize at the higher temperature also crystallizes. Equation for the stress in the polymer can be shown to be given by Eq.(38) and it can be solved for the change in stretch during the crystallization. Crystallization rates are identical to the ones used earlier and can be given by Eq.(36) and Eq(37). The solution procedure for melting-1 is similar to TSCP-1 with the difference that the stress in the polymer is not zero during the process. The melting rate used is identical to the one used earlier and can be given by Eq.(45). Stress in the polymer can be shown to be given by Eq.(43); this equation can be solved for the change in stretch during the melting process. During melting2 , the polymer is heated above the first recovery temperature and the first phase that crystallized both at the higher and the lower temperatures melts, the polymer returns to the original deformed shape. The equation for stress in the polymer can be shown to be given by Eq.(47), with the difference that the stress 
in the material is not zero. Melting rates used are identical to Eq.(49) and Eq.(50). The variation of stretch with time is calculated in a manner similar to TSCP-1.

\subsection{TSCP-4}

This is a one-step triple shape creation procedure, here the triple shape effect is observed during recovery. This TSCP consists of five phases Loading, Crystallization, Unloading, Melting-1 and Melting-2. Equations for each stage of this TSCP can be derived using a similar methodology as used during the previous cases. During the loading process stress in the material can be shown to be given by Eq.(24). During crystallization the stress is maintained constant and the polymer is cooled to a temperature below both the recovery temperatures. On cooling below the first recovery temperature the first phase crystallizes, on further cooling below the second recovery temperature the second phase crystallizes, along with a portion of the first phase that crystallizes at the lower temperature. Using similar methodology as used for the previous cases the stress in the material can be shown to be given by Eq.(38). The rate equations used are identical to Eq.(27), Eq.(36) and Eq.(37). Upon unloading the first temporary shape is fixed, the stress in the material can be shown to be given by Eq.(40). During melting-1 the polymer is heated above the second recovery temperature, the second phase melts and the polymer reverts to an intermediate shape between the first temporary shape and the permanent shape as the material is a mixture of amorphous phase and the first phase which crystallized both at the higher and lower temperatures. This is the second temporary shape, thus a triple shape effect is observed during recovery. The stress can be shown to be given by Eq.(43), the melting rate equation used is identical to Eq.(45). During melting-2 the polymer is heated above the second recovery temperature, the first phase crystallized both at the higher and lower temperatures melts and the polymer reverts back to the original shape. The stress can be shown to be given by Eq.(47); the melting rate equations used are identical to Eq.(49) and Eq.(50). The variation of stretch with time is determined in a manner identical to TSCP-1.

\section{Results}

In this section, we will discuss results for triple shape effect and different TSCPs, generated from the execution of calculations described in the previous section. The calculations have been carried out for geometry of uni-axial extension undergoing deformation using four different triple shape creation procedures. We have also studied two types of results, crystallization under constant stress and crystallization under constant strain. In addition the results of the calculation for the simulation of TSCP3 are compared against experimental data.

The first set of results is for TSCP-1 with the crystallization processes both at the higher and lower temperatures taking place while the stress is kept constant. The graph of true stress versus stretch ratio is shown in Fig.5. Note that the stress increases when the polymer is deformed above both the recovery temperatures. During crystallization- 1 at the higher temperature crystalline phase- 1 is formed in a stress free state, for the stress to be held constant, the polymer will have to immediately undergo a small extension, thus as crystallization continues the material extends. Upon unloading-1 there is a small retraction as the new material is significantly stiffer than the original amorphous material. As the material is stiffer the stress increases sharply as the polymer is deformed during loading-2. During crystallization2 two phases are formed phase- 2 and phase- 1 that crystallizes at lower temperature, again as the crystalline phases are formed in a stress free state, for the stress to be held constant the polymer will have to undergo a small extension. As the material is lot stiffer here the extension is smaller as compared to 
previous case. Upon unloading-2 the polymer again retracts by a small but finite amount because of the increase in stiffness. Fig.6 shows the graph of stress versus stretch for TSCP-1 for crystallization under constant stress for different values of final crystallinity of phase-1 that cyrystallizes at higher temperature. Note that as the final crystallinity in the material increases the stiffness also increases, this can be detected by looking at the slopes of the curves during unloading-1 and loading- 2 .

We also looked at the case in TSCP-1 when the crystallization takes place while the strain is kept constant. This case is shown in Fig.7, note that after the onset of crystallization-1 the stress drops as the newly formed crystalline material i.e. phase- 1 at higher temperature is formed in a stress free state. After unloading- 1 the sample retracts by a small amount because of increase in stiffness. During loading-2 as can be seen from the slope of the curve, the material is a lot stiffer as compared to amorphous state during loading-1. After the onset of crystallization-2, there is again a drop in stress as the newly formed crystalline phases are formed in a stress free state. Upon unloading-2, there is again a small retraction due to increase in stiffness after the crystallization process. For TSCP-1 with crystallization under constant strain the graph for stress versus stretch for different values of final crystallinities of phase- 1 crystallized at higher temperature is shown in Fig.8. Note that the drop in stress observed during crystallization increases for larger final values of crystallinity. Also, the results clearly show that greater the crystallinity, greater the stiffness.

The next case studied is TSCP-2, with the crystallization processes at the higher and lower temperatures both taking place while the stress is kept constant, which is shown in Fig.9. As the polymer is deformed above both the recovery temperatures the stress increases. As the crystalline phases are formed in a stress free state, the polymer will have to undergo a small extension for stress to be held constant during crystallization. After this the polymer is unloaded. This can be observed in Fig.9. During the first heating above the second recovery temperature i.e. melting-1, there is a drop in stretch as the crystals of phase- 2 that contribute to fixation of temporary shape melt and return to amorphous state. This is how the first temporary shape (B) is obtained. Because of this, the two loops overlap unlike what is seen in TSCP-1. As can be seen by the slope of the curve, the material is a lot stiffer during loading-2 due to the presence of crystalline phase- 1 crystallized both at the higher and the lower temperatures. During crystallization-2 as the crystalline phase (phase-2) is formed in a stress free state the material extends as the crystallization proceeds. On unloading- 2 there is again a small retraction, and the second temporary shape (A) is fixed completely by the crystallites of phase-2. The first cooling process to $T_{L}$ allows for complete crystallization of phase-1, at both higher and lower temperatures. As a complete crystallization of phase1 has been allowed during first cooling process, it does not crystallize during the second cooling to temperature $T_{L}$. Consequently, the fixation of second temporary shape (A) is limited to the crystallites of phase-2. As the second temporary shape is fixed only by phase-2, TSCP-2 allows for better recovery from second temporary shape to the first temporary shape as compared to TSCP-1.

For TSCP-2, we also looked at the case when crystallization takes place while the strain is kept constant. This case is shown in Fig.10, the stress increases as the polymer is deformed above recovery temperature. As explained earlier on the onset of crystallization-1 the stress drops. Again, due to the melting of phase2 we see the two loops overlap unlike what is seen in TSCP-1. During loading-2 the material is stiffer due to presence of crystalline phases as can be seen from the slope of the curve. Again, after the onset of 
crystallization during crystallization-2 there is a drop in stress. There is a small retraction upon unloading- 2 as the material is stiffer after crystallization.

The third case we investigated was TSCP-3 for uni-axial extension. Fig.11 shows the variation of stretch with time throughout the deformation cycle for this case, three sets of curves are plotted, each corresponding to a different level of final crystallinity of phase- 1 crystallized at higher temperature. The polymer is deformed prior to the cooling cycle this causes an initial deformation in the polymer before the start of the crystallization process. This initial stress is maintained upon the polymer throughout the triple shape creation cycle. On cooling below the first recovery temperature phase-1 crystallizes, as the crystalline phase is formed in a stress-free state, for the stress to be held constant the polymer will have to immediately undergo an extension. This causes the fixation of first temporary shape above the second recovery temperature. On further cooling the polymer undergoes further crystallization and consequently a further extension, as the material is a lot stiffer due to previous crystallization the extension is small. During cooling-2, phase2- crystallizes along with part of phase-1 that crystallizes at lower temperature. This causes the fixation of second temporary shape. On heating above the second recovery temperature phase- 2 melts this causes a drop in the stretch, as the crystalline phase responsible to maintain the stretch transforms back to the amorphous phase. Note, that phase-1 crystallized at lower temperature also contributes to the fixation of the second temporary shape, which does not melt at this point; as a result the first temporary shape is not completely recovered, i.e., there is an asymmetry in the curve because of asymmetry in the crystallization and melting process. On further heating above the first recovery temperature, phase- 1 that crystallized both at the higher and lower temperature melts, and the polymer returns to the original stretch. Note that as the final crystallinity of phase-1 increases, the TSMP stretches more as more of the crystalline phase is formed in a stress free state and the polymer has to extend to maintain the constant stress.

For TSCP-3, we compare the behavior of the model with data presented in Zotzmann et al. [40]. For the data presented the material does not exhibit viscoelastic behavior or creep. Note, the time range used for the triple shape cycle is long, this is due to the low rate of cooling and heating used to ensure complete polymer crystallization and melting. The stiffness material constants were chosen to be realistic for this kind of polymer, and the crystallization kinetics based on the crystallization data given in [40]. The plot for stretch versus time is shown in Fig.12. As you can see the data points compare very well with the model calculations, i.e., they capture the trend. However, it should be noted that this does not purport to be a prediction, though it does show that the model can capture the overall trend. Fitting the model to a specific polymer will require an independent set of experiments to fit the material constants and a different set of data to compare the predictions against. Such a comprehensive set of experimental data is not yet available for these new materials and data is still scarce. Also note that in this case due to the composition of TSMP, the amount of first phase crystallized at a higher temperature is insufficient to cause an increase in the stretch. During melting, first phase crystallized at a lower temperature does not melt at the lower recovery temperature thus preventing complete recovery and causes the second recovery step at the higher recovery temperature (for details see Zotzmann et al. [40]). Therefore a triple shape effect can be observed during the heating process.

The next case we investigate is the one step triple shape creation procedure or TSCP-4. Fig.13 shows the stretch versus time plot during the entire deformation cycle, for this case three sets of curves are plotted each corresponding to a different amount of final crystallinity of phase- 1 crystallized at higher 
temperature. Note, that the stretch increases when the polymer is deformed above both the recovery temperatures. During the cooling process the polymer is cooled to a temperature below both the transition temperatures. During the initial part of the cooling at a temperature above the second transition temperature phase- 1 crystallizes, and the polymer undergoes an extension. In the later part of cooling process below the second transition temperature phase- 2 crystallizes along with part of phase- 1 that crystallizes at lower temperature and material undergoes a slight extension. Upon unloading there is a slight retraction due to increase in stiffness as a result of crystallization. On heating above the second recovery temperature phase- 2 melts and the polymer retracts to an intermediate shape or the second temporary shape, as phase- 2 transforms to amorphous state. On further heating above the first transition temperature phase-1 formed both at the higher and lower temperatures melts and the polymer retracts to its original shape. Note that as the amount of final crystallinity of phase-1 increases the stretch undergone by the polymer for the same reasons discussed above.

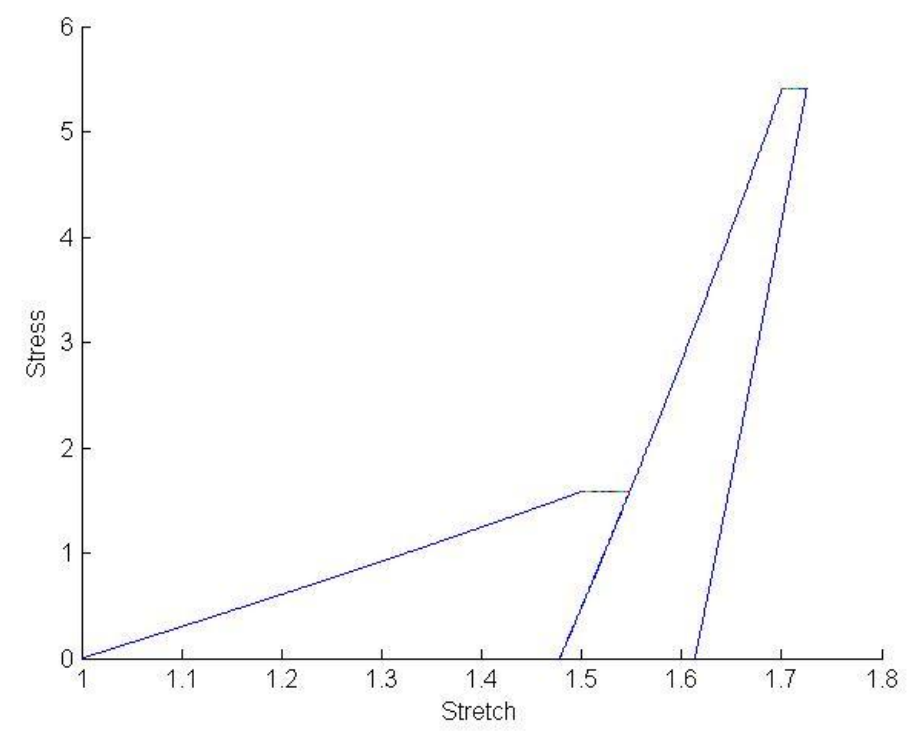

Figure 5. Plot of stress versus stretch for uni-axial extension with crystallization taking place at constant stress during TSCP-1. . The constants used for the simulation are $\mu_{a}=0.78 \mathrm{Mpa}, \mu_{1}=25, \mu_{2}=15$, $\mu_{4}=25, \mu_{5}=15, G_{1}=0.00007 s^{-2}, G_{2}=0.00007 s^{-2}, \alpha_{1_{H}}=0.4, \alpha_{1_{L}}=0.2, \alpha_{2}=0.2, t_{1}=50$, $t_{3}=600, t_{5}=1150, t_{7}=1800$. 


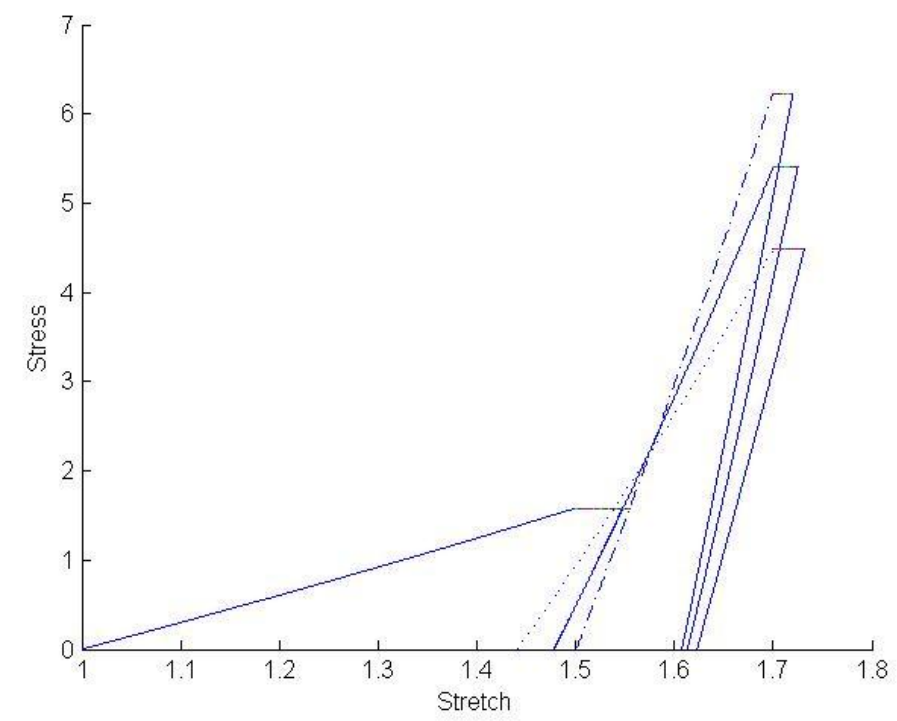

Figure 6. Plot of stress versus stretch for uni-axial extension with crystallization taking place at constant stress during TSCP-1 for three different crystallinities of phase- 1 crystallized at higher temperature. The constants used for the simulation are $\mu_{a}=0.78 \mathrm{Mpa}, \mu_{1}=25, \mu_{2}=15, \quad \mu_{4}=25, \mu_{5}=15$, $G_{1}=0.00007 s^{-2}, G_{2}=0.00007 s^{-2}, \alpha_{1_{L}}=0.2, \alpha_{2}=0.2, t_{1}=50, t_{3}=600, t_{5}=1150, t_{7}=1800$. $\left(\ldots \ldots \alpha_{1_{H}}=0.2, \ldots \alpha_{1_{H}}=0.3, \ldots .-. .-\alpha_{1_{H}}=0.4,\right)$

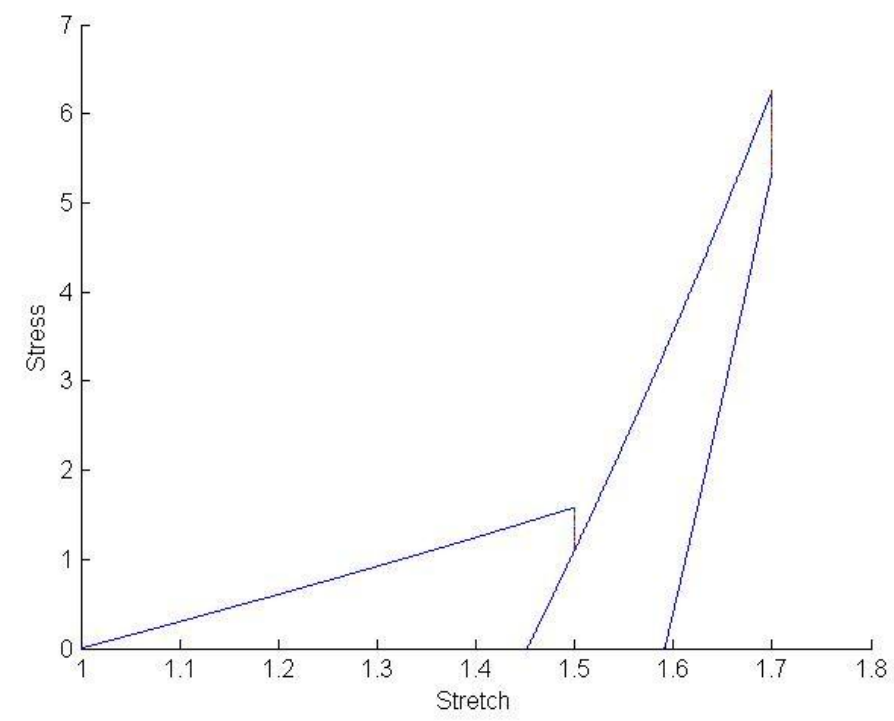

Figure 7. Plot of stress versus stretch for uni-axial extension with crystallization taking place at constant strain during TSCP-1. The constants used for the simulation are $\mu_{a}=0.78 M p a, \mu_{1}=25, \mu_{2}=15$, $\mu_{4}=25, \mu_{5}=15, G_{1}=0.00007 s^{-2}, \quad G_{2}=0.00007 s^{-2}, \quad \alpha_{1_{H}}=0.4, \quad \alpha_{1_{L}}=0.2, \alpha_{2}=0.2, t_{1}=50$, $t_{3}=600, t_{5}=1150, t_{7}=1800$. 


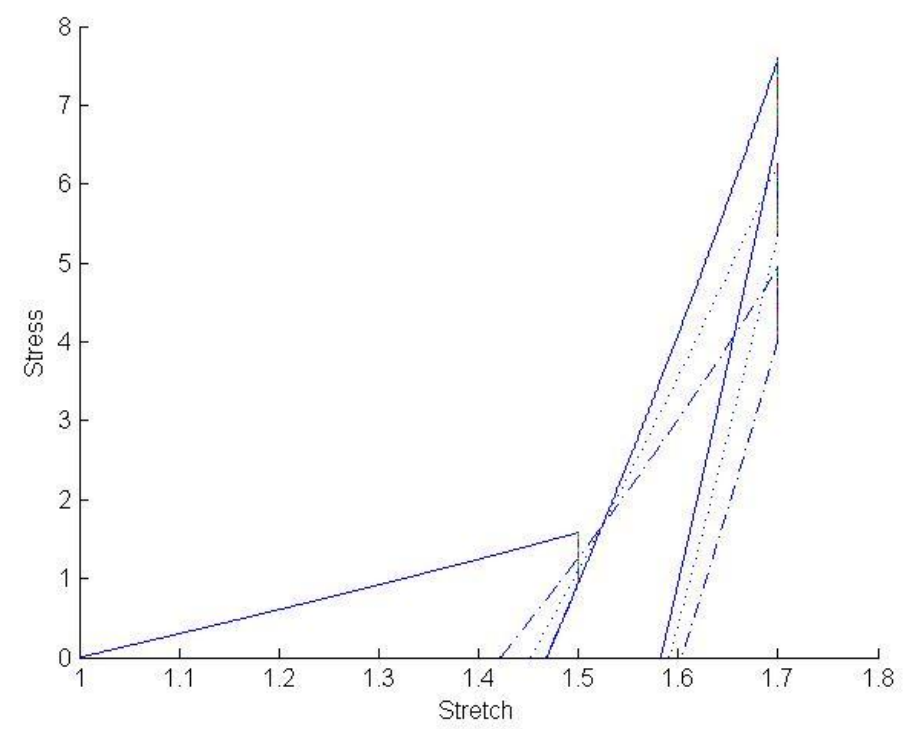

Figure 8. Plot of stress versus stretch for uni-axial extension with crystallization taking place at constant strain during TSCP-1 for three different crystallinities of phase-1 crystallized at higher temperature. The constants used for the simulation are $\mu_{a}=0.78 \mathrm{Mpa}, \mu_{1}=25, \mu_{2}=15, \quad \mu_{4}=25, \mu_{5}=15$, $G_{1}=0.00007 s^{-2}, G_{2}=0.00007 s^{-2}, \alpha_{1_{L}}=0.2, \alpha_{2}=0.2, t_{1}=50, t_{3}=600, t_{5}=1150, t_{7}=1800$. $\left(\ldots \ldots \alpha_{1_{H}}=0.2, \ldots \alpha_{1_{H}}=0.3, \ldots . . .-\alpha_{1_{H}}=0.4,\right)$

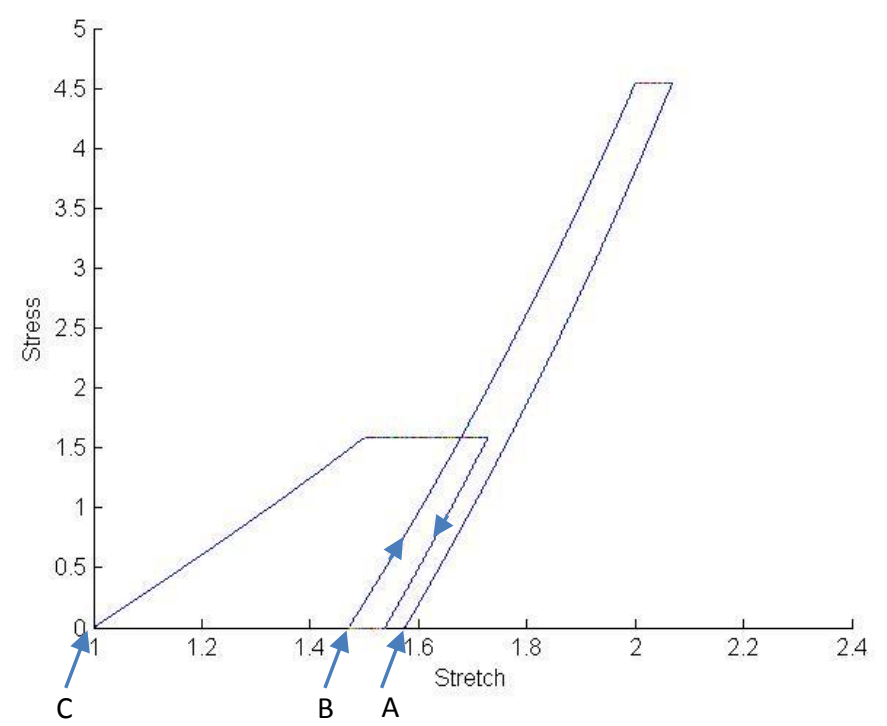

Figure 9. Plot of stress versus stretch for uni-axial extension with crystallization taking place at constant stress during TSCP-2. The constants used for the simulation are $\mu_{a}=0.78 \mathrm{Mpa}, \mu_{1}=5, \mu_{2}=2$, $\mu_{4}=2.5, \mu_{5}=1.2, G_{1}=0.00007 s^{-2}, G_{2}=0.00007 s^{-2}, \alpha_{1_{H}}=0.15, \alpha_{1_{L}}=0.2, \alpha_{2}=0.2, t_{1}=50$, $t_{3}=600, t_{5}=1150, t_{7}=1800$. 


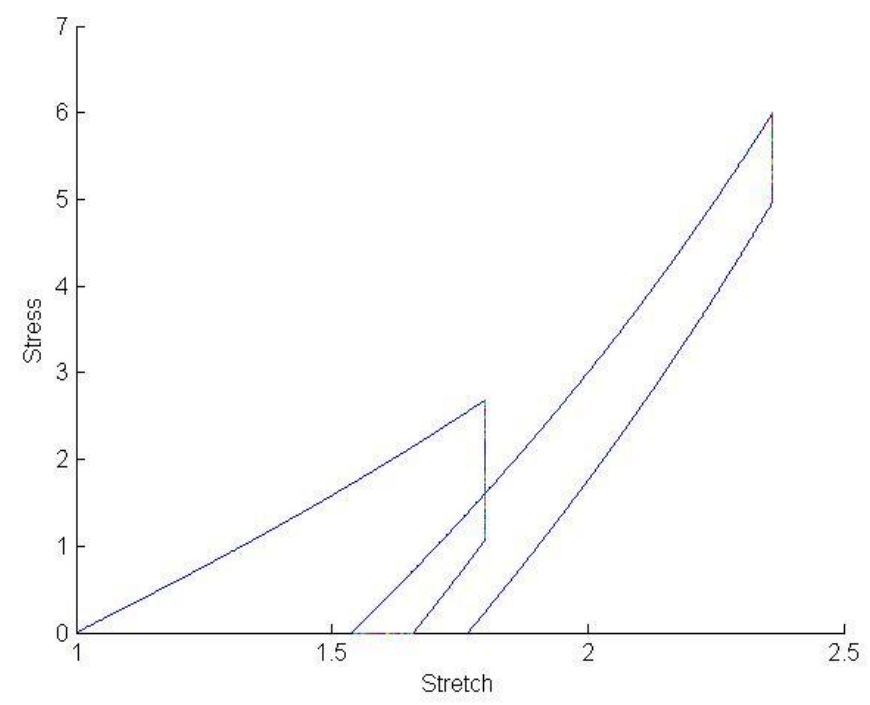

Figure 10. Plot of stress versus stretch for uni-axial extension with crystallization taking place at constant stress during TSCP-2. The constants used for the simulation are $\mu_{a}=0.78 \mathrm{Mpa}, \mu_{1}=5, \mu_{2}=2$, $\mu_{4}=2.5, \mu_{5}=1.2, G_{1}=0.00007 \mathrm{~s}^{-2}, G_{2}=0.00007 \mathrm{~s}^{-2}, \alpha_{1_{H}}=0.15, \alpha_{1_{L}}=0.2, \alpha_{2}=0.2, t_{1}=50$, $t_{3}=600, t_{5}=1150, t_{7}=1800$.

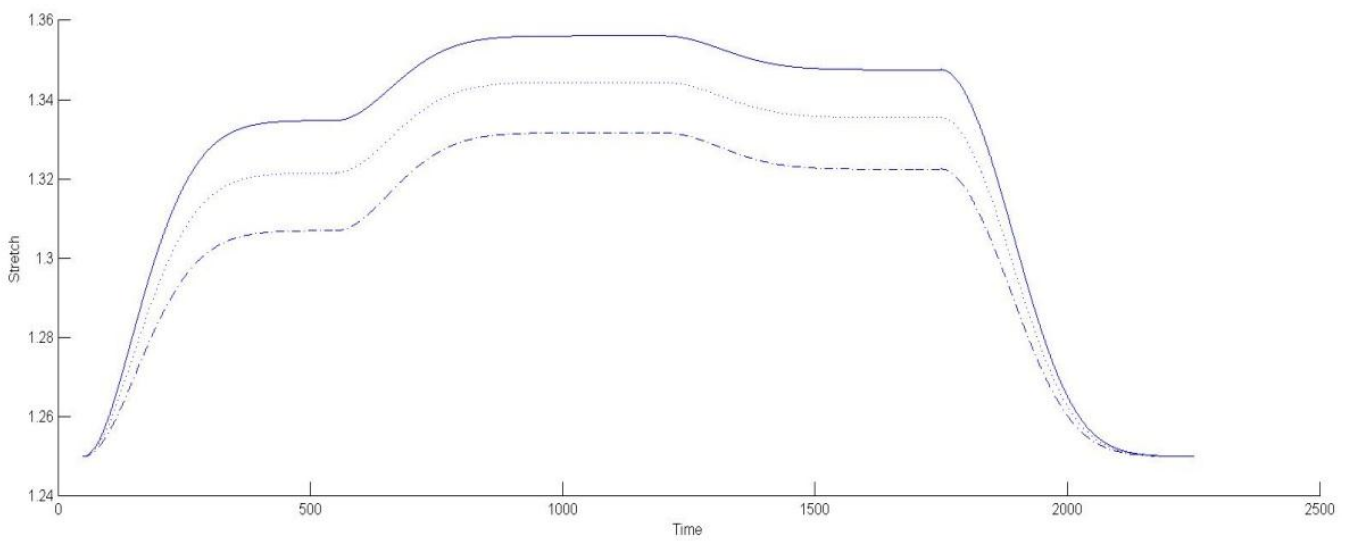

Figure 11. Plot of stretch versus time for uni-axial extension with crystallization taking place at constant stress during TSCP-3 for three different values crystallinities of phase-1 crystallized at higher temperature. The constants used for the simulation are $\mu_{a}=0.78 \mathrm{Mpa}, \mu_{1}=3.3, \mu_{2}=3, \mu_{4}=10$, $\mu_{5}=6, G_{1}=0.00007 s^{-2}, G_{2}=0.00007 s^{-2}, \alpha_{1_{H}}=0.3, \alpha_{1_{L}}=0.1, \alpha_{2}=0.1, t_{1}=50, t_{3}=600$, $t_{5}=1150, t_{7}=1800 .\left(\_\alpha_{1_{H}}=0.5, \ldots \ldots \alpha_{1_{H}}=0.4, \ldots . . .-\alpha_{1_{H}}=0.3,\right)$ 


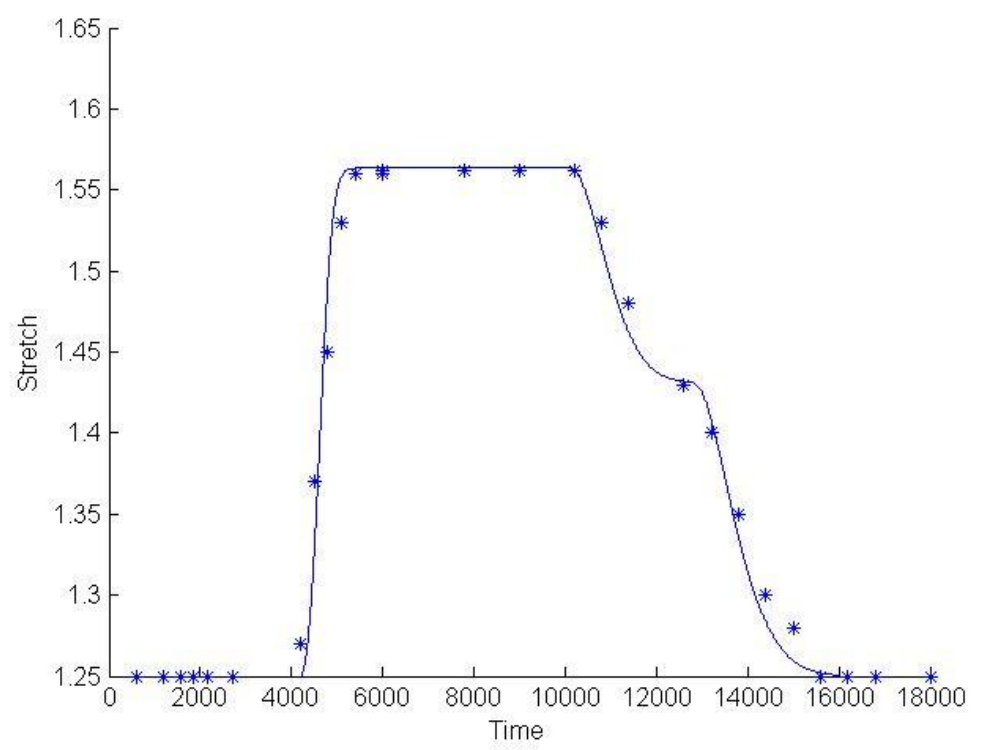

Figure 12. Plot of stretch versus time for uni-axial extension with crystallization taking place at constant stress during TSCP-3. *indicates experimental data (Zotzmann et al.). The constants used for the simulation are $\mu_{a}=0.78 \mathrm{Mpa}, \mu_{1}=0.06, \mu_{2}=0.04, \quad \mu_{4}=0.06, \mu_{5}=0.04, G_{1}=0.000012 \mathrm{~s}^{-2}$, $G_{2}=0.000012 s^{-2}, \alpha_{1_{H}}=0, \alpha_{1_{L}}=0.1, \alpha_{2}=0.1, t_{1}=4200, t_{3}=10100, t_{7}=12800$.

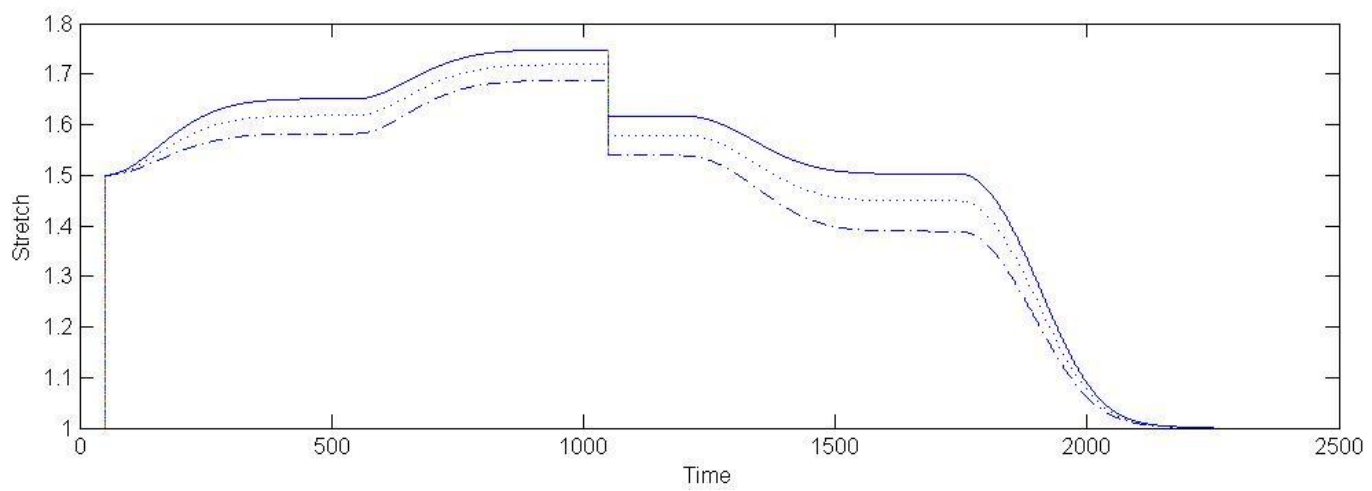

Figure 13. Plot of stretch versus time for uni-axial extension with crystallization taking place at constant stress during TSCP-4 for three different values crystallinities of phase-1 crystallized at higher temperature. The constants used for the simulation are $\mu_{a}=0.78 \mathrm{Mpa}, \mu_{1}=3.3, \mu_{2}=3, \mu_{4}=10$, $\mu_{5}=6, G_{1}=0.00007 \mathrm{~s}^{-2}, G_{2}=0.00007 \mathrm{~s}^{-2}, \alpha_{1_{L}}=0.2, \alpha_{2}=0.2, t_{1}=50, t_{3}=600, t_{5}=1200$, $t_{7}=1750$. $\alpha_{1_{H}}=0.4, \ldots \ldots \alpha_{1_{H}}=0.3,-. .-.-\alpha_{1_{H}}=0.2$, ) 


\section{Conclusion}

In this work a constitutive model has been developed to describe the mechanical behavior of crystallizable triple shape memory polymers. The theory of multiple natural configurations has been used to develop the models. The model has been solved for a uni-axial extension deformation cycle. A key part of this work is the investigation of diverse triple shape creation procedures. Each shape memory creation process results in a different triple shape memory cycle. This variety of shape memory creation processes and the resulting different shape memory cycles is a key feature of this material. It is envisaged that this flexibility can be exploited to find use in many different applications. The results have been compared with experimental data and show good agreement. Here, it should be noted that the models presented here have been developed in a mechanical setting. However, in order to study the full scope of the problem, thermodynamics to tie in heat transfer and rate equations for crystallization and melting will have to be used. This analysis is currently under development. It is anticipated that this work will contribute to the development of reliable constitutive models that will lead to a better and more in-depth understanding of triple shape memory polymers and thus to continued innovation in the application and development in a wide array of technologies for which these materials are well suited.

\section{ACKNOWLEDGMENTS}

The authors would like to thank National Science Foundation for supporting this work under grant number 0900278.

\section{References}

[1] R. J. Atkins and R. E. Craine, Continuum theory of mixtures: basic theory and historical development. Quarterly Journal of Mechanics and Applied Mathematics, 29 (1976), 209-244.

[2] R. M. Bowen, Theory of mixtures, in Continuum Physics Vol. III, A. C. Eringen, ed., Academic Press New York 1975, 1-127.

[3] C. Eckart, The thermodynamics of irreversible processes. IV. The theory of elasticity and anelasticity. 73 (1948), 373-382.

[4] M. Irie, in Shape Memory Materials, K. Otsuka and C. M. Wayman, eds., Cambridge University Press, Cambridge 1998, 203-219.

[5] H. M. Jeong, B. K. Kim and Y. J. Choi, Synthesis and properties of thermotropic liquid crystalline polyurethane elastomer. Polymer 41 (2000), 1849-1855.

[6] B. K. Kim, S.Y. Lee and M. Xu, Polyurethanes having shape memory effects. Polymer 37 (1996), 5781-5793.

[7] B. K. Kim,S. Y. Lee, J. S. Lee, S. H. Baek, Y. J. Choi, J. O. Lee and M. Xu, Polyurethane ionomers having shape memory effects. Polymer 39 (1998), 2803-2808. 
[8] A. Lendlein, A. M. Schmidt and R. Langer, AB-polymer networks based on oligo (e-caprolactone) segments showing shape memory properties. Proceedings of the National Academy of Sciences , USA,98, (2001), 842-847.

[9] A. Lendlien and S. Kelch, Shape memory polymers. Angewandte Chemie International Edition 41(2002), 2034-2057.

[10] F. Li, X. Zhang, J. Hou, M. Xu, X. Luo, D. Ma and B. K. Kim, Studies on thermally stimulated shape memory effect of segmented polyurethanes. Journal of Applied Polymer Science 64 (1997), 15111516.

[11] A. Lendlein, Advances in Polymer Science, Shape-Memory Polymers. 226, Springer, New York 2010 .

[12] J. E. Mark, Interpretation of polymer properties in terms of chain conformations and spatial configurations. Acc. Chem. Res. 12 (1979), 49-55.

[13] J. E. Mark, B. Erman, Rubberlike Elasticity: A Molecular Primer. Wiley Interscience, New York 1988.

[14] G. J. Monkman, Advances in Shape Memory Actuation. Mechatronics 10 (2000), 489-498.

[15] R. W. Ogden, Non-Linear Elastic Deformations. Dover, NY 1984.

[16] C. Poliane, P. Delobelle, C. Lexcellent, S. Hayashi and H. Tobushi, Analysis of the mechanical behavior of shape memory polymer membranes by nanoindentation, bulging and point membrane detection tests. Thin Solid Films 379 (2000), 156-165.

[17] K. R. Rajagopal and A. S. Wineman, A constitutive equation for non-linear solids which undergo deformation induced microstructural changes. International Journal of Plasticity 8 (1992), 385-395.

[18] K. R. Rajagopal, Multiple configurations in continuum mechanics. Reports of the Institute for Computational and Applied Mechanics, University of Pittsburgh, 6 (1995).

[19] K. R. Rajagopal and A. R. Srinivasa, On the inelastic behavior of solids: Part-1-Twinning. International Journal of Plasticity. 11 (1995), 653-678.

[20] K. R. Rajagopal and L. Tao, Mechanics of Mixtures. World Scientific, River Edge, NJ 1995.

[21] K. R. Rajagopal and A. R. Srinivasa, Inelastic behavior of solids: Part-1-Theoretical under-pinning. International Journal of Plasticity 14 (1998), 945-967.

[22] K. R. Rajagopal and A. R. Srinivasa, On the thermodynamics of shape memory wires. ZAMP 50 (1999), 459-496.

[23] K. R. Rajagopal and A. R. Srinivasa, A Thermodynamic frame-work for rate type fluid models, Journal of Non-Newtonian Fluid Mechanics 88 (2000), 207-227. 
[24] I. J. Rao and K. R. Rajagopal, Phenomenological modeling of crystallization in polymers using the notion of multiple natural configurations. Intefaces and Boundaries 2 (2000), 73-94.

[25] I. J. Rao and K. R. Rajagopal, A study of strain induced crystallization in polymers. International Journal of Solids and Structures 38 (2001), 1149-1167.

[26] I. J. Rao, Constitutive modeling of crystallizable shape memory polymers. Proceedings of SPEANTEC, San Francisco 2002.

[27] I. J. Rao and K. R. Rajagopal, A thermodynamic framework for the study of crystallization in polymers. ZAMP 53 (2002), 365-406.

[28] I. J. Rao, Effect of the rate of deformation on the crystallization behavior of polymers. International Journal of Non-Linear Mechanics 38 (2003), 663-676.

[29] I. J. Rao, J. D. Humphrey and K. R. Rajagopal, Biological growth and remodeling: a uni-axial example with possible application to tendons and ligaments. Computer Modeling in Engineering and Science 4 (2003), 439-456.

[30] I. J. Rao and K. R. Rajagopal, Simulation of the film blowing process for semi-crystalline polymers. Modeling and Simulation of Materials 12 (2005), 129-146.

[31] I. J. Rao and K. R. Rajagopal, On the modeling of quiescent crystallization of polymer melts. Polymer Engineering and Science 44 (2004), 123-130.

[32] W. G. Reyntjens, F. E. Du Prez and E. J. Geothals, Polymer networks containing crystallizable poly(octadecyl vinyl ether) segments for shape-memory materials. Macromolecular Rapid Communications 20 (1999), 251-255.

[33] S. J. Tey, W. M. Huang and W. M. Sokolowski, Influence of long-term storage in cold hibernation on strain recovery and recovery stress of polyurethane shape memory foam. Smart Materials and Structures 10 (2001), 321-325.

[34] H. Tobushi, H. Hara, E. Yamada and S. Hayashi, Thermomechanical properties in a thin film of a shape memory polymer of polurethane series, Smart Materials and Structures. 5 (1996), 483-491.

[35] M. Serrano, A. Guillermo, Recent insights into the biomedical applications of Shape-memory Polymers, Macromolecular Bioscience 12 (2012), 1156-1171.

[36] A. Lendlein, M. Behl, B. Hiebl, C. Wischke, Shape-memory polymers as a technology platform for biomedical applications, Expert Reviews 7(3) (2010), 357-379.

[37] L. R. G. Treolar, The Physics of Rubber Elasticity, 3rd edn., Oxford University Press, 1975.

[38] C. Truesdell, Sulle basi della thermomeccanica, Rendicontie Lincei. 22 (1957), 33-38.

[39] M. Wang and L. Zhang, Recovery as a measure of oriented crystalline structure in poly(etherester)s based on poly(ethylene oxide) and poly(ethylene terephthalate) used as shape memory polymers, Journal of Polymer Science, Part B 37 (1999), 101-112. 
[40] J.Zotzmann, Marc Behl, Dieter Hofmann, and A. Lendlein, Reversible Triple-Shape Effect of Polymer Networks Containing Polypentadecalactone and Poly (e-caprolactone)- Segments. Advanced Materials, 22 (2010), 3424-3429.

[41] J.Zotzmann, Marc Behl, Yakai Feng, and A. Lendlein, Copolymer Networks Based on Poly (wpemtadeclactone) and Poly(e-caprolactone) Segments as a Versatile Triple-Shape Polymer System. Advanced Functional Materials. 20 (2010), 3583-3594.

[42] J.Zotzmann, Marc Behl, and A. Lendlein, The Influence of Programming Conditions on the TripleShape Effect of Copolymer Networks with Poly(w-pentadecalactone) and Poly(e-caprolactone) as Switching Segments. Marcomolecule Symposium. 309/310 (2011), 147-153.

[43] G. Barot and I.J. Rao, Constitutive modeling of the mechanics associated with crystallizable shape memory polymers. Zietschrift fur angewandte Methematik und Physik ZAMP. 57 (2006), 652-681.

[44] G. Barot, I.J. Rao, and K.R. Rajagopal, A thermodynamic framework for the modeling of crystallizable shape memory polymers. Journal of Engineering Science. 46 (2008), 325-351.

[45] M. Behl and A. Lendlein, Triple-shape Polymers. Journal of Material Chemistry. 20 (2012), 33323334.

[46] I. Bellin, S. Kelch, R. Langer, and A. Lendlein, Polymeric triple-shape materials. 103 (2006), 1804318047.

[47] P.T. Mather, X. Luo, and I, Rousseau, Shape Memory Polymer Research. Annual Review Material Research. 39 (2009), 445-471.

[48] L. Sun, W.M. Wang, Z. Ding, Y. Zhao, C.C. Wang, H. Purnawali, C. Tang, Stimulus-responsive shape memory materials: A review. Journal of Material Design, 33 (2012), 577-640.

[49] J. S. Sodhi, I. J. Rao, Modeling the mechanics of light activated shape memory polymers. International journal of engineering science. 48 (2010), 1576-1589.

[50] T. Xie, Recent advances in polymer shape memory. Polymer, 52 (2011), 4985-5000.

[51] J. Hu, H. Meng, G. Li, S. I. Ibekwe, A review of stimuli-responsive polymers for smart textile applications. Smart Mater. Struct. 21 (2012), 053001 (23pp).

[52] J. Hu, Y. Zhu, H. Huang, J. Lu, Recent advances in shape-memory polymers: Structure, mechanism, functionality, modeling and applications. Progress in polymer science. 37 (2012), 1720-1763.

[53] J. Leng, X. Lan, Y. Liu, S. Du, Shape-memory polymers and their composites: Stimulus methods and applications. Progress in Material Science 56 (2011), 1077-1135.

[54] Q. Zhao, M. Behl, A. Lendlein, Shape-memory polymers with multiple transitions: complex actively moving polymers. Soft Matter 9 (2013), 1744-1755.

[55] A. Sisson, A. Lendlein, Advances in actively moving polymers. Macromolecular Materials and Engineering. 297 (2012), 1135-1137. 
[56] M. Heuchel, T. Sauter, K. Kratz, A. Lendlein, Thermally induced shape-memory effects in polymers: Quantification and related modeling approaches. Polymer Physics. 51 (2013), 621-637. 